\title{
Modeling Intercity Travel Mode Choice with Data Balance Changes: A Comparative Analysis of Bayesian Logit Model and Artificial Neural Networks
}

\author{
Xiaowei Li $\mathbb{D}^{1},{ }^{1}$ Yuting Wang, ${ }^{1}$ Yao Wu $\mathbb{D}^{2},{ }^{2}$ Jun Chen, ${ }^{1}$ and Jibiao Zhou $\mathbb{D}^{3}$ \\ ${ }^{1}$ School of Civil Engineering, Xi'an University of Architecture \& Technology, Xi'an 710055, China \\ ${ }^{2}$ School of Modern Posts and Institute of Modern Posts, Nanjing University of Posts and Telecommunications, \\ Nanjing 210003, China \\ ${ }^{3}$ College of Transportation Engineering, Tongji University, Shanghai 201804, China
}

Correspondence should be addressed to Yao Wu; wuyao@njupt.edu.cn

Received 1 July 2021; Revised 26 August 2021; Accepted 1 September 2021; Published 14 September 2021

Academic Editor: Xiangyang Guan

Copyright (c) 2021 Xiaowei Li et al. This is an open access article distributed under the Creative Commons Attribution License, which permits unrestricted use, distribution, and reproduction in any medium, provided the original work is properly cited.

\begin{abstract}
This study conducts a comprehensive comparative analysis of regression-based multinomial models and artificial neural network models in intercity travel mode choices. The four intercity travel modes of airplane, high-speed rail (HSR), train, and express bus were used for analysis. Passengers' activity data over the process of intercity travel were collected to develop the models. The standard multinomial logit (MNL) regression and Bayesian multinomial logit (BMNL) regression were compared with the radial basis function (RBF) and multilayer perceptron (MLP). The results show that MLP performs best in terms of predictive accuracy, followed by BMNL and MNL, and RBF is the least accurate. The performances of all models were examined against changes in data balance, and it was found that rebalancing can improve fitting performance while slightly reducing the predictive performance. This comparative study and its parameter estimation shed new light on the comparison of traditional and emerging models in travel behavior studies, and the findings can be used as heuristic guidance for all stakeholders.
\end{abstract}

\section{Introduction}

To model passengers' travel behaviors is of value to better understand mobility modes in the complex travel environment [1]. Policies and managerial strategies rely on the accurate estimation of travel mode choices of passengers. In 2020, COVID-19 has profoundly influenced passengers' travel behaviors, causing a dramatic shift in intracity and intercity mobility modes, inevitably affecting society, production, and the global economy. Scholars have investigated contextual factors that influence travel modes, aiming to better understand passengers' choices and develop suitable models.

Previous studies have shown that travel mode choice can be affected by social and demographic factors, including gender [2-4], age [4-6], occupation [3], income [2, 4, 7, 8], and car ownership [4]. Miskeen et al. [4] found that males were more likely to use public transportation than cars, while females were less likely to shift to public transportation. Cheng et al. [6] indicated that age was the most significant individual-related attribute. Tourists were more likely to choose a plane or train than a coach [3]. Forinash et al. [7] found that high- and low-income groups preferred air travel and bus, respectively. It was similarly reported that an increase in passengers' incomes decreased their use of buses [4]. Lower-income individuals were found to be more sensitive to cost and less sensitive to out-of-vehicle time than middle- and high-income individuals [8]. Related attributes, such as travel demand, service quality of transport modes, and accessibility of transportation hubs, have been found to influence travel mode choices $[1,3,9,10]$.

The most widely used modeling techniques in travel mode choice are discrete choice models, such as the binomial logit (BL) [11], multinomial logit (MNL) [4, 12], 
multinomial probit (MNP) [3], nested logit (NL) [13, 14], and mixed logit (ML) models [15-17], which have high interpretability of estimation results on input variables, as well as high transferability and validity. Regression-based models form maximum likelihood estimates of parameters $[4,5,11,12,17,18]$. Apart from the popular logit model, Bayesian parameter estimation methods have shown good accuracy and performance [19-22]. For example, Wong and Farooq [23] developed an algorithm based on the restricted Boltzmann machine, which has multiple discrete-continuous layers and can be expressed as a variational Bayesian inference optimization problem.

Emerging machine learning techniques have been studied for travel mode choice [24-34]. Lindner et al. [33] found an artificial neural network (ANN) and classification tree $(\mathrm{CT})$ to outperform binary logit regression in motorized travel mode choice. Cheng et al. [6] found the random forest (RF) to have significantly better prediction accuracy than support vector machine (SVM), adaptive boosting (AdaBoost), and MNL in modeling travel mode choice. Zhao et al. [24] compared the model development, evaluation, and behavior interpretation of MNL and ML with that of the naive Bayes, CT, AdaBoost, bag fruit tree, RF, SVM, and ANN machine learning classifiers. Among machine learning approaches, the multilayer perceptron (MLP) and radial basis function (RBF) have been widely applied due to their better classification accuracy compared to naive Bayes, K-nearest neighbors, and backpropagation neural networks [35-37]. Hence, they have potential use in the study of travel mode choice.

The influence of data balance on the accuracy of multimode choice models has not been widely reported. Imbalanced sample data can influence the accuracy of estimation in multiclass discrete choice prediction $[38,39]$, and methods such as oversampling and undersampling have been proposed to address this issue $[40,41]$. However, there is no commonly agreed best method to resolve this issue in multiclass classification. This is a well-known issue in travel mode choice, and the effectiveness of rebalancing methods when using different regression-based and neural network models in empirical studies of modeling travel mode choice requires study.

This study has three objectives: (1) to investigate the predictive performance of modeling techniques including Bayesian multinomial logit (BMNL), MNL, MLP, and RBF for intercity travel mode choice; (2) to assess the predictive performance of the above techniques after data balancing; and (3) to evaluate the factors affecting intercity travel mode choice and their relative importance using a comprehensive dataset. Passengers' activity data over the whole process of intercity travel were collected. The travel modes of airplane, HSR, train, and express bus were investigated. The BMNL, MNL, MLP, and RBF models were developed and validated. A receiver operating characteristic (ROC) curve and confusion matrix were employed to evaluate the models' predictive performance.

The remainder of this paper is organized as follows. Section 2 introduces the methodological background of the selected models, followed by a description of the dataset in Section 3. Section 4 presents the results and findings. We summarize our conclusions and propose future work in Section 5 .

\section{Methodology}

2.1. Bayesian Multinomial Logit Model. MNL regression generalizes logistic regression into multiclass problems that consist of more than two possible discrete groups $[1,19]$. It can be expressed as [19]

$$
P\left(Z_{j}=i\right)=\frac{\exp \left(\beta_{0}^{i}+\beta_{1}^{i} x_{j 1}+\beta_{2}^{i} x_{j 2}+\cdots+\beta_{k}^{i} x_{j k}\right)}{\sum_{i=1}^{I} \exp \left(\beta_{0}^{i}+\beta_{1}^{i} x_{j 1}+\beta_{2}^{i} x_{j 2}+\cdots+\beta_{k}^{i} x_{j k}\right)},
$$

where $\mathbf{X}=\left[x_{j 1}, x_{j 2}, \ldots, x_{j k}\right]$ is a vector of independent variables $x_{j k}, \beta=\left[\beta_{0}^{i}, \beta_{1}^{i}, \beta_{2}^{i}, \ldots, \beta_{k}^{i}\right]^{T}$ is the corresponding coefficient vector, and $Z_{j}=i$ is the choice of travel mode $i$ for the $j^{\text {th }}$ observation.

The likelihood function can be expressed as

$$
\begin{aligned}
f(\mathbf{Z} \mid \boldsymbol{\beta}) & =\prod_{j=1}^{N} \prod_{i=1}^{I}\left[\varepsilon_{j i} \times P\left(Z_{j}=i\right)\right] \\
& =\prod_{j=1}^{N} \prod_{i=1}^{I}\left[\varepsilon_{j i} \times \frac{\exp \left(\beta_{0}^{i}+\beta_{1}^{i} x_{j 1}+\beta_{2}^{i} x_{j 2}+\cdots+\beta_{k}^{i} x_{j k}\right)}{\sum_{m=1}^{I} \exp \left(\beta_{0}^{i}+\beta_{1}^{i} x_{j 1}+\beta_{2}^{i} x_{j 2}+\cdots+\beta_{k}^{i} x_{j k}\right)}\right],
\end{aligned}
$$

where $N$ is the number of samples, $I$ is the number of outcomes, and $\varepsilon_{i j}$ equals 1 when the discrete outcome of sample $j$ is $i$ and is 0 otherwise.

The Bayesian approach using Markov chain Monte Carlo (MCMC) was utilized for model estimation. Based on Bayesian inference, the posterior joint distribution of parameters $\beta$ conditional on dataset $\mathbf{Z}$ can be estimated as [19]

$$
f(\boldsymbol{\beta} \mid \mathbf{Z})=\frac{f(\mathbf{Z}, \boldsymbol{\beta})}{f(\mathbf{Z})}=\frac{f(\mathbf{Z} \mid \boldsymbol{\beta}) \pi(\boldsymbol{\beta})}{f(\mathbf{Z} \mid \boldsymbol{\beta}) \pi(\boldsymbol{\beta}) d(\boldsymbol{\beta})} \propto f(\mathbf{Z} \mid \boldsymbol{\beta}) \pi(\boldsymbol{\beta}),
$$

where $f(\mathbf{Z}, \beta)$ is the joint probability distribution of $\mathbf{Z}$ and $\beta$, $f(\mathbf{Z} \mid \beta)$ is the likelihood of the conditional function based on $\beta$, and $\pi(\beta)$ is the prior distribution of $\beta$. Due to lack of information on the random parameters, we used the noninformative prior distributions [1]: 


$$
\boldsymbol{\beta} \sim N\left(0_{k}, 10^{6} M_{k}\right),
$$

where $0_{k}$ is a vector of zeros and $M_{k}$ is the $k \times k$ identity matrix.

The posterior joint distribution can be derived as [42]

$$
\begin{aligned}
f(\boldsymbol{\beta} \mid \mathbf{Z}) \propto f(\mathbf{Z} \mid \boldsymbol{\beta}) \pi(\boldsymbol{\beta})= & \prod_{j=1}^{N} \prod_{i=1}^{I}\left[\varepsilon_{j i} \times \frac{\exp \left(\beta_{0}^{i}+\beta_{1}^{i} x_{j 1}+\beta_{2}^{i} x_{j 2}+\cdots+\beta_{k}^{i} x_{j k}\right)}{\sum_{m=1}^{I} \exp \left(\beta_{0}^{i}+\beta_{1}^{i} x_{j 1}+\beta_{2}^{i} x_{j 2}+\cdots+\beta_{k}^{i} x_{j k}\right)}\right] \\
& \times \prod_{j=1}^{N} \prod_{i=1}^{I}\left[\frac{1}{\sqrt{2 \pi} 10^{3}} \exp \left(-\frac{1}{2} \frac{\left(\beta_{k}^{i}\right)^{2}}{10^{6}}\right)\right] \propto \exp \left\{\sum_{j=1}^{N} \sum_{i=1}^{I}\left[\varepsilon_{i j} \times \frac{\exp \left(\beta_{0}^{i}+\beta_{1}^{i} x_{j 1}+\beta_{2}^{i} x_{j 2}+\cdots+\beta_{k}^{i} x_{j k}\right)}{\sum_{m=1}^{I} \exp \left(\beta_{0}^{i}+\beta_{1}^{i} x_{j 1}+\beta_{2}^{i} x_{j 2}+\cdots+\beta_{k}^{i} x_{j k}\right)}\right]-\sum_{j=1}^{N} \sum_{i=1}^{I}\left[\frac{1}{2} \frac{\left(\beta_{k}^{i}\right)^{2}}{10^{6}}\right]\right\} .
\end{aligned}
$$

\subsection{Radial Basis Function (RBF) Neural Network. An RBF} neural network is a typical three-layer neural network model with input, hidden, and output layers, as shown in Figure 1, where $k$ is the number of input variables, $H$ is the number of hidden neurons, $I$ is the number of output neurons (travel modes), $\mathbf{X}=\left[x_{1}, x_{2}, \ldots, x_{k}\right]^{T}$ is the input, $\mathbf{Y}=\left[y_{1}, y_{2}, \ldots, y_{I}\right]^{T}$ is the output, and $w_{h i}$ is the connection weight of the $h^{\text {th }}$ hidden layer neuron to the $i^{\text {th }}$ output layer neuron.

A Gaussian function is generally used as the hidden layer excitation function. The output of the $h^{\text {th }}$ hidden layer neuron is

$$
G_{h}(x)=e^{\left(-X-c_{h}^{2} / 2 \sigma_{h}^{2}\right)}, \quad h=1,2, \ldots, H,
$$

and the linear mapping relationship between $G_{h}(x)$ and the $i^{\text {th }}$ output layer neuron is

$$
G_{h}(x)=y_{i}=\sum_{h=1}^{H} w_{h i} G_{h}(x), \quad i=1,2, \ldots, I,
$$

where $c_{h}$ and $\sigma_{h}$ are, respectively, the center vector of the Gaussian function and the base width of the $h^{\text {th }}$ hidden neuron.

RBF has been criticized as a "black box" that lacks interpretability [43]. Various tools have been developed to address this issue, the most common being variable importance analysis [44-46], which measures the relative importance of each independent variable in predicting dependent variables.

2.3. Multilayer Perceptron (MLP) Neural Network. MLP is a commonly used supervised ANN model that can be used for both pattern recognition and function approximation. Compared to RBF, MLP can have multiple hidden layers (shown in Figure 2) [47]. The hyperbolic tangent function is selected as the activation function of MLP hidden neurons. The output from a hidden neuron is

$$
y=\frac{e^{u}-e^{-u}}{e^{u}+e^{-u}}
$$

and the connection weight is the output of the net function,

$$
u=b+\sum_{p=1}^{k} w_{p} x_{p}
$$

where $k$ is the number of inputs, $x_{p}$ is the input, $w_{p}$ is the weight of the corresponding input $\left(w_{p} ; 1 \leq p \leq k\right), b$ is the bias weight, and the Levenberg-Marquardt training algorithm is selected [28].

2.4. Model Comparison and Validation. The multiclassification confusion matrix (see Table 1) is used to calculate the accuracy of each model [48], where $s_{i m}$ is the number of samples in which mode $i$ is predicted as mode $m$. The recall and precision of mode $i$ are

$$
\begin{aligned}
\text { Recall }_{i} & =\frac{S_{i i}}{\sum_{m=1}^{I} s_{i m}}, \\
\text { Precision }_{i} & =\frac{S_{i i}}{\sum_{m=1}^{I} S_{m i}},
\end{aligned}
$$

and the accuracy of the model can be calculated as [1]

$$
\text { accuracy }=\frac{\sum_{i=1}^{I} S_{i i}}{N}
$$

The ROC curve and area under the curve (AUC) were also used to measure the predictive ability. A higher AUC value indicates better predictive accuracy $[42,49]$.

\section{Data Collection}

Data from Li et al.'s work [42] were used in this study. A total of 985 random samples collected in Xi'an from March 1-10, 2018, were used for analysis, where 161 samples reported the choice of airplane, accounting for $16.3 \%$ of intercity travel records, and 369 (37.5\%) were reported as HSR, 299 (30.4\%) as train, and $156(15.8 \%)$ as express bus. Among them, 80\% were randomly selected for training, and the remaining $20 \%$ were used for prediction. In addition to the original information included in the database, the travel distance was calculated by Baidu Maps using the real route between the cities of origin and destination. The intercity travel time was obtained according to the identification number of the carrier, transportation schedule, and origin and destination cities.

Undersampling and oversampling are the most frequently used techniques to balance data for machine learning and pattern recognition [38-41]. Undersampling 


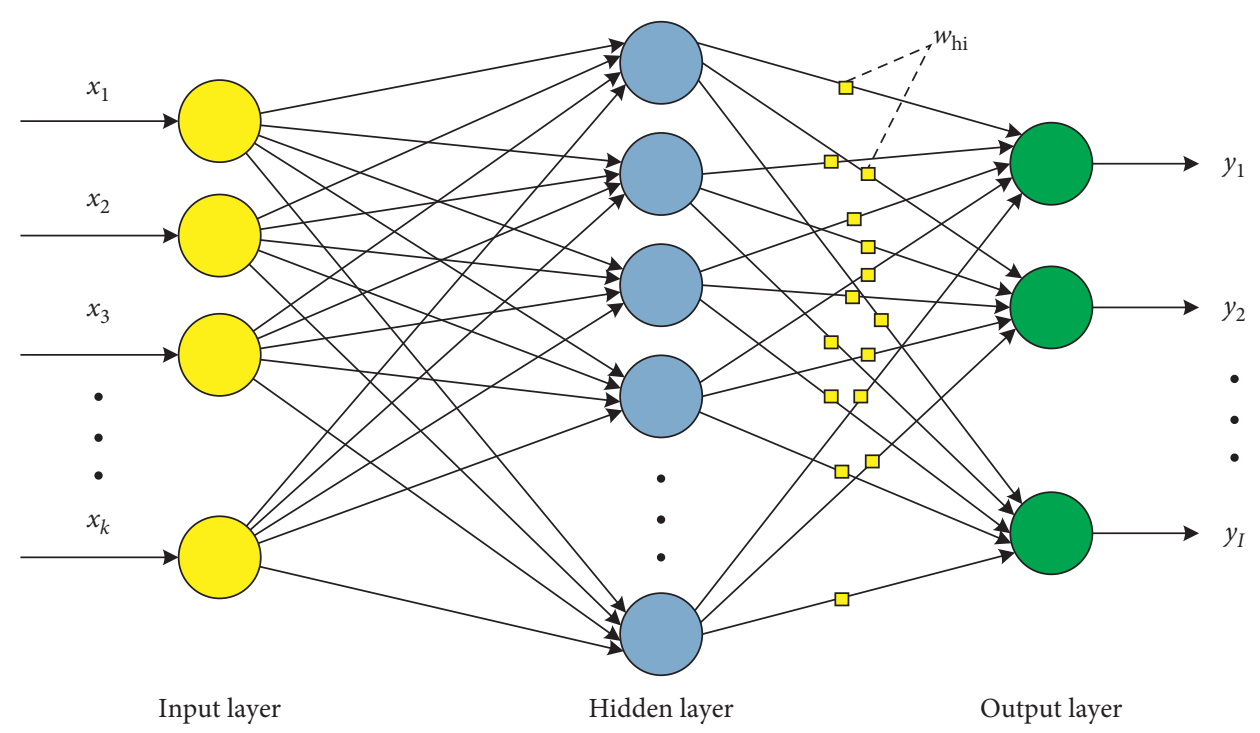

Figure 1: RBF network.

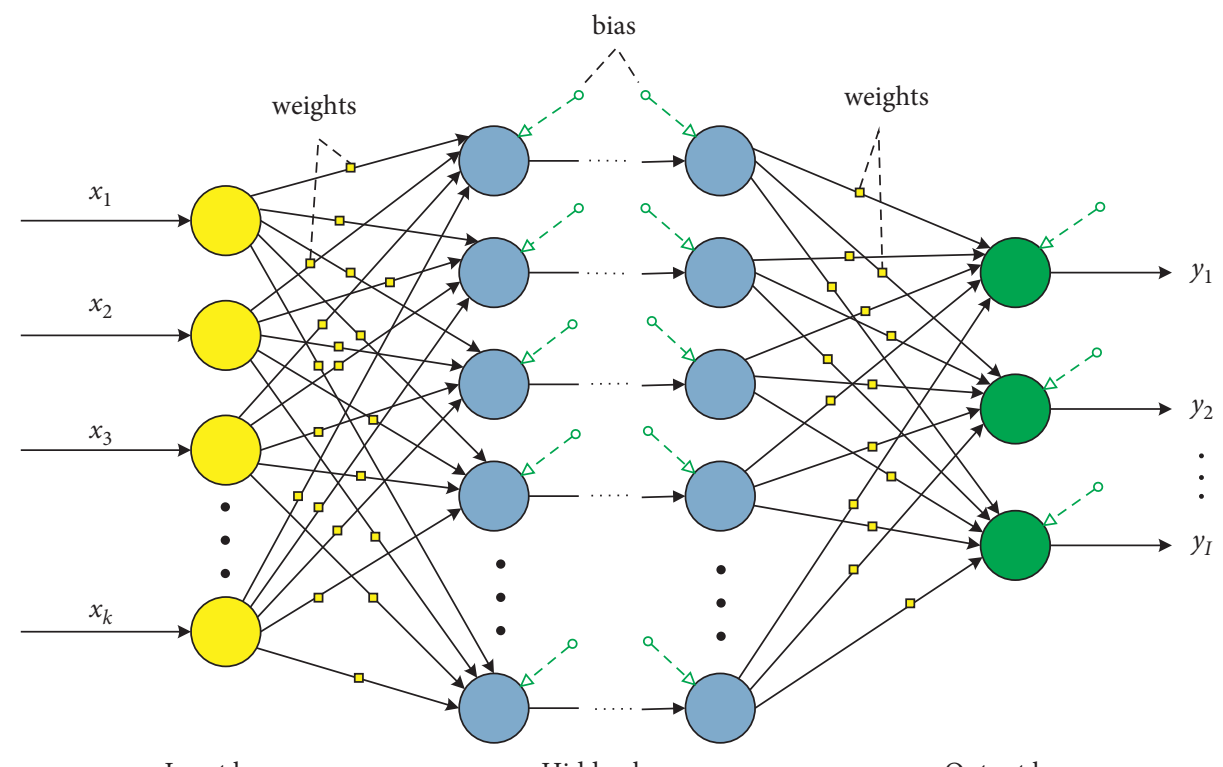

Input layer

Hidden layer

Output layer

Figure 2: General topology of MLP.

TABLE 1: Multiclassification confusion matrix.

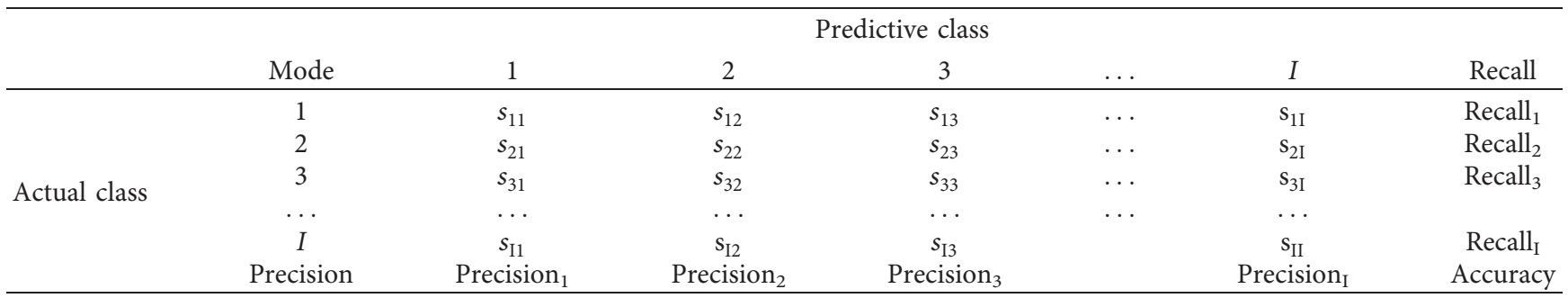

achieves relative equilibrium among classes by reducing the number of samples of classes with more samples. Using this method, the number of samples of each travel mode was 156, with $80 \%$ randomly selected for training, and the remaining $20 \%$ selected for prediction. Oversampling is to add samples of classes with fewer samples to equal the number of samples 
in a class with more samples. Through oversampling, the sample size of each transportation mode became $369 ; 80 \%$ of samples were randomly selected for training, and the remaining $20 \%$ were selected for prediction.

Tables 2 and 3 describe the categories and continuous variables for imbalanced and rebalanced data, respectively.

\section{Results}

Stata 15.0 software was used for parameter estimation of the BMNL and MNL models, confusion matrix, and ROCs. SPSS 25.0 was used for relative importance analysis of variables by the RBF and MLP models.

4.1. Model Results. Table 4 presents the estimated means of variables from the BMNL model, and Tables 5-7 show their parameter estimates. The frequently used train was considered as the reference in the model. The typical variables including gender, age, occupation, travel purpose, monthly income, intercity travel distance, intercity travel cost, intercity travel time, safety, comfort, punctuality, access time, and departure time were selected for modeling after collinearity testing. The MCMC simulation-based full Bayesian approach was employed to estimate the posterior distributions of parameters. Variables with confidence intervals not including zero were regarded as significant [19]. As shown in Table 4, we found that the parameter estimates of certain variables differed slightly between the imbalanced and balanced data. For example, the intercity travel distance is significantly related to the choice of express bus when using balanced data, but not when using imbalanced data. The signs of variables were found to be consistent between balanced and imbalanced data.

Table 8 shows the estimated coefficients of variables from the MNL model using the same variables. Parameter estimates of variables are shown in Tables 9-11. Similar to the BMNL model, the parameter estimates differ to some extent between imbalanced and balanced data, and the signs of significant variables are consistent. The symbols of significant variables in the MNL model were consistent with those in the BMNL model. However, the significant variables in MNL were not completely consistent with those in the BMNL model. For example, the travel purpose is significant in the BMNL model but not in the MNL model. Gender was significantly related to the choice of HSR in the BMNL model, but not in the MNL model.

Figures 3 and 4 show the relative importance of the factors obtained by RBF and MLP, respectively. There is a slight difference in the order of relative importance of factors. For example, using imbalanced data, intercity travel cost is most important in the RBF model, but second in importance in the MLP model, after intercity travel time. Slight differences exist in the relative importance of factors between imbalanced and balanced data in the same model. For example, in the MLP model, gender is the least important using imbalanced data, and travel purpose is the least important using balanced data. Overall, intercity travel cost, intercity travel time, intercity travel distance, comfort, safety, and punctuality are the most important factors in the intercity travel mode choice, followed by the monthly income, age, and occupation. Access and departure times, which reflect the accessibility of a transport hub, show moderate importance. Travel purpose and gender are the least important.

\subsection{Model Comparison and Validation}

4.2.1. Model Performance for Imbalanced Data. AUCs and confusion matrices were employed to compare the fitting and predictive performance of the MNL, BMNL, MLP, and RBF models. The confusion matrix of the four models using imbalanced data is shown in Table 12. Through the analysis of the accuracy, it can be found that MLP has the best fitting performance $(80.70 \%)$, and $\mathrm{RBF}$ is the worst $(67.30 \%)$. BMNL (76.36\%) and MNL (76.10\%) have similar fitting performance. For the predictive set, the predictive performance of MLP (78.70\%) is the best, followed by MNL (75.76\%), BMNL (75.25\%), and RBF (65.50\%).

The ROC curves of the four models are shown in Figures 5 and 6. For the training set, the AUC of the MLP for the airplane is 0.9857 , which indicates that its fitting performance is better than that of BMNL (0.9732), MNL (0.9731), and RBF (0.9443). The MLP model is almost perfect, as its ROC curve rises rapidly toward the upper-left corner of the graph. Similarly, the AUCs of MLP for HSR and train are the largest, followed by BMNL, MNL, and RBF. These findings confirm that the MLP model outperforms BMNL and MNL, followed by RBF.

For the predictive set, the AUC of MLP for airplane is 0.9905, which is better than RBF (0.9823), BMNL (0.9784), and MNL (0.9767). Similarly, MLP is almost perfect, as its ROC curve rises rapidly toward the upper-left corner of the graph. The AUC of MLP for HSR is also the largest, followed by BMNL, MNL, and RBF. The AUC of MLP for train is 0.9054 , which indicates that its predictive performance is significantly better than that of MNL (0.8637), BMNL (0.8624), and RBF (0.8280). However, the AUC of BMNL for express bus is the largest, followed by MNL, MLP, and RBF.

4.2.2. Model Performance for Rebalanced Data. MNL, BMNL, MLP, and RBF were used to train and verify the balanced data with the same variables used for the imbalanced data. The confusion matrices of each model for undersampled and oversampled balanced data are shown in Tables 13 and 14. The ROC curves for the undersampled training and predictive set are presented in Figures 7 and 8. The ROC curves for the oversampled training and predictive sets are presented in Figures 9 and 10.

The results show that MLP provides the best fitting for both oversampled and undersampled data, followed by BMNL and MNL, and RBF has the poorest fitting performance. The results are consistent with those of the four models using imbalanced data. Hence, whether the data are balanced will not affect the relative fitting performance of the models. 


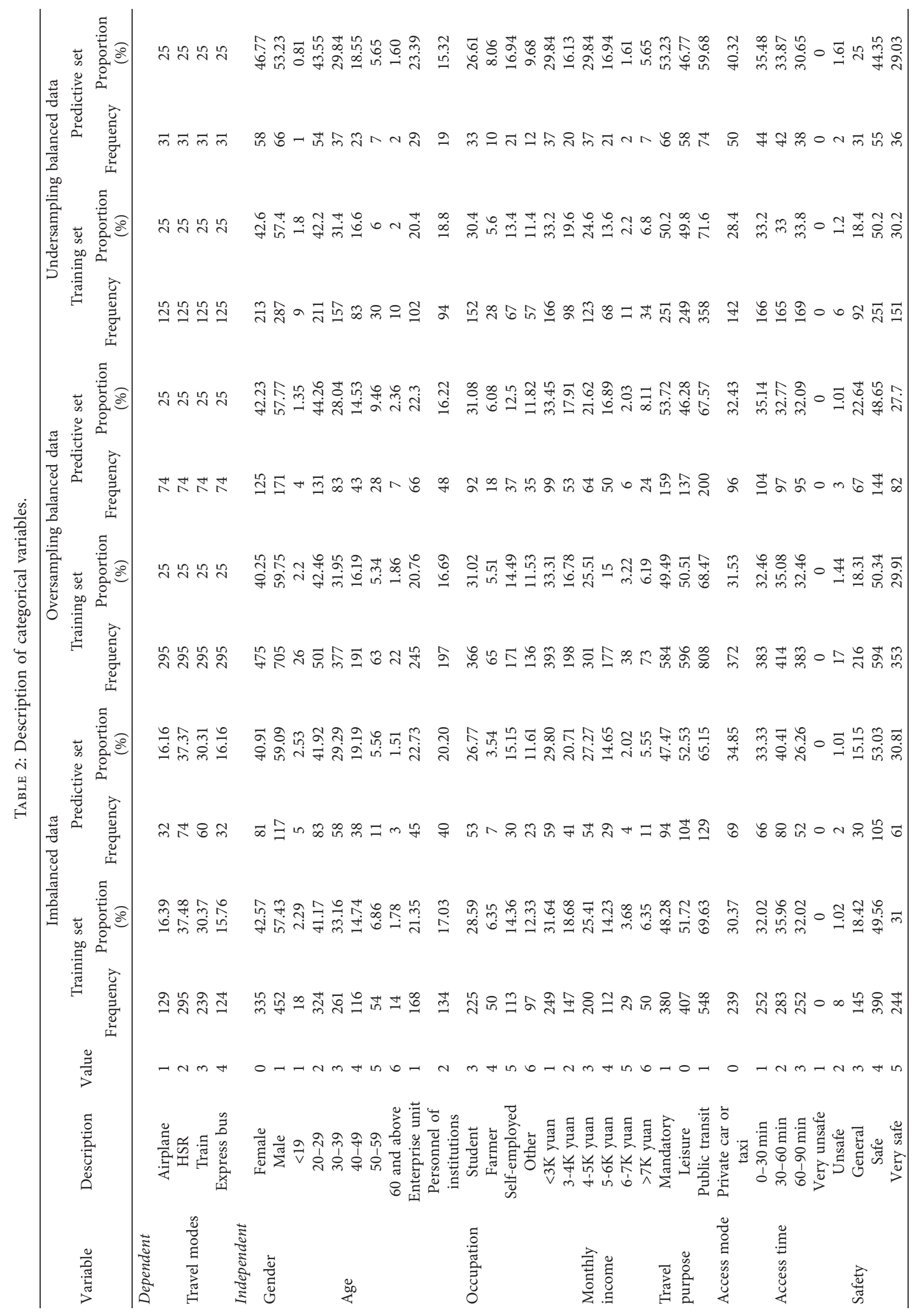




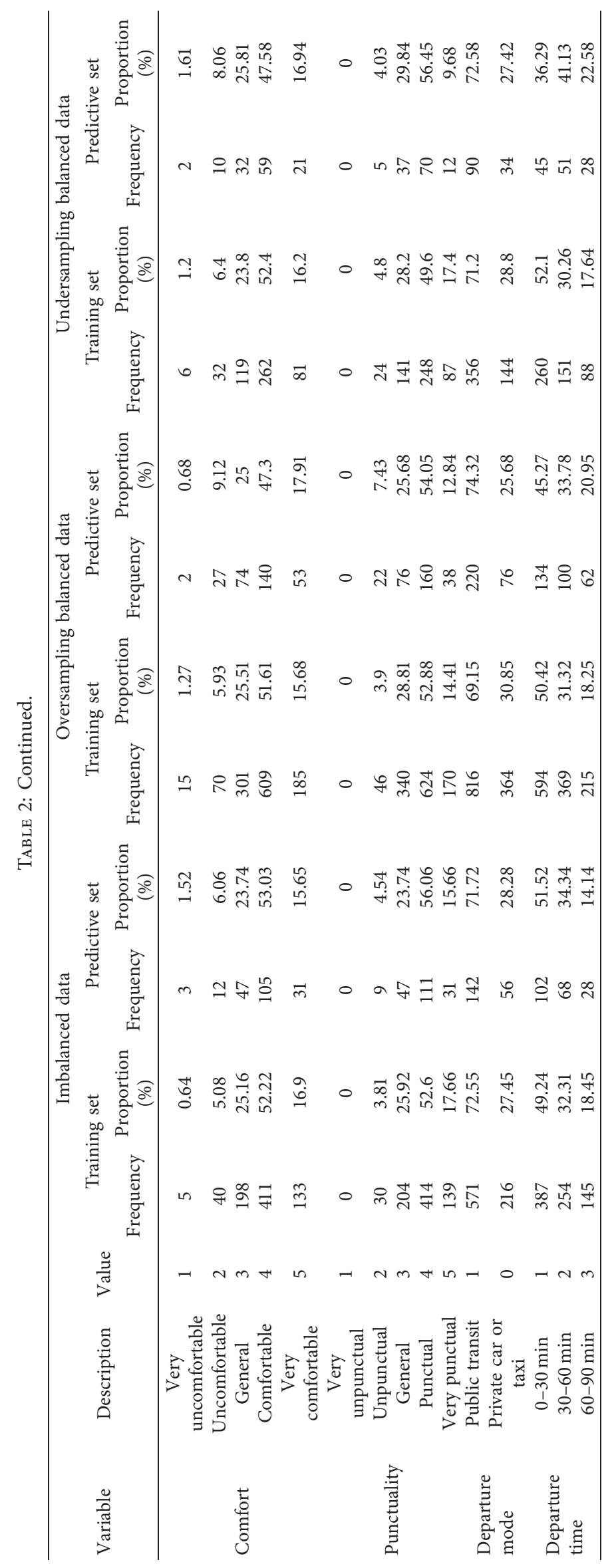


TABLE 3: Description of continuous variables.

\begin{tabular}{|c|c|c|c|c|c|c|c|c|c|c|}
\hline \multirow{2}{*}{ Data } & \multirow{2}{*}{ Variable } & \multirow{2}{*}{ Unit } & \multicolumn{4}{|c|}{ Training set } & \multicolumn{4}{|c|}{ Predictive set } \\
\hline & & & Min & Max & Mean & $\mathrm{SD}$ & Min & Max & Mean & SD \\
\hline \multirow{5}{*}{ Imbalanced data } & Intercity travel distance & $\mathrm{km}$ & 16.00 & 2831.00 & 797.54 & 579.86 & 16.00 & 2540.00 & 792.66 & 632.00 \\
\hline & Intercity travel cost & Yuan & 7.00 & 2600.00 & 310.46 & 319.44 & 7.00 & 1400.00 & 283.07 & 288.70 \\
\hline & Intercity travel time & Hour & 0.22 & 52.00 & 6.48 & 6.49 & 0.25 & 33.00 & 6.00 & 5.87 \\
\hline & Access cost & Yuan & 1.00 & 300.00 & 11.59 & 22.89 & 1.00 & 100.00 & 10.19 & 16.36 \\
\hline & Departure cost & Yuan & 1.00 & 150.00 & 13.52 & 20.72 & 1.00 & 150.00 & 13.23 & 21.89 \\
\hline \multirow{5}{*}{ Oversampling balanced data } & Intercity travel distance & $\mathrm{Km}$ & 16.00 & 2831.00 & 807.54 & 621.35 & 16.00 & 2801.00 & 808.45 & 617.20 \\
\hline & Intercity travel cost & Yuan & 7.00 & 2600.00 & 321.23 & 343.35 & 7.00 & 2600.00 & 324.57 & 344.48 \\
\hline & Intercity travel time & Hour & 0.22 & 52.00 & 5.92 & 6.33 & 0.25 & 37.00 & 5.85 & 6.50 \\
\hline & Access cost & Yuan & 1.00 & 300.00 & 13.03 & 25.51 & 1.00 & 150.00 & 11.57 & 20.38 \\
\hline & Departure cost & Yuan & 1.00 & 150.00 & 15.19 & 23.36 & 1.00 & 150.00 & 14.24 & 22.69 \\
\hline \multirow{5}{*}{ Undersampling balanced data } & Intercity travel distance & $\mathrm{Km}$ & 17.00 & 2831.00 & 823.55 & 616.21 & 27.00 & 2500.00 & 731.52 & 597.32 \\
\hline & Intercity travel cost & Yuan & 7.00 & 2600.00 & 330.79 & 360.54 & 13.50 & 1200.00 & 277.20 & 275.22 \\
\hline & Intercity travel time & Hour & 0.22 & 37.00 & 5.92 & 6.14 & 0.25 & 28.00 & 5.22 & 5.90 \\
\hline & Access cost & Yuan & 1.00 & 300.00 & 12.77 & 25.43 & 1.00 & 120.00 & 12.49 & 19.88 \\
\hline & Departure cost & Yuan & 1.00 & 150.00 & 14.68 & 23.11 & 1.00 & 120.00 & 14.51 & 22.79 \\
\hline
\end{tabular}

TABle 4: Parameter estimation in BMNL.

\begin{tabular}{|c|c|c|c|c|c|c|c|c|c|}
\hline \multirow[b]{2}{*}{ Variable } & \multicolumn{3}{|c|}{ Imbalanced data } & \multicolumn{3}{|c|}{ Oversampling balanced data } & \multicolumn{3}{|c|}{ Undersampling balanced data } \\
\hline & $\begin{array}{c}\text { Airplane } \\
\text { Mean }\end{array}$ & $\begin{array}{l}\text { HSR } \\
\text { Mean }\end{array}$ & $\begin{array}{c}\text { Express bus } \\
\text { Mean }\end{array}$ & $\begin{array}{c}\text { Airplane } \\
\text { Mean }\end{array}$ & $\begin{array}{l}\text { HSR } \\
\text { Mean }\end{array}$ & $\begin{array}{c}\text { Express bus } \\
\text { Mean }\end{array}$ & $\begin{array}{l}\text { Airplane } \\
\text { Mean }\end{array}$ & $\begin{array}{c}\text { HSR } \\
\text { Mean }\end{array}$ & $\begin{array}{c}\text { Express bus } \\
\text { Mean }\end{array}$ \\
\hline \multicolumn{10}{|l|}{ Gender } \\
\hline Male vs. female & 0.555 & 0.186 & 0.368 & 0.683 & 0.197 & 0.713 & 1.231 & 0.384 & 0.733 \\
\hline Age & 0.282 & 0.350 & 0.247 & 0.416 & 0.357 & 0.346 & 0.396 & - & 0.380 \\
\hline \multicolumn{10}{|l|}{ Occupation } \\
\hline $\begin{array}{l}\text { Personnel of institutions vs. } \\
\text { enterprise unit }\end{array}$ & 0.710 & 0.408 & 0.651 & 0.344 & 0.143 & 0.313 & - & - & - \\
\hline Student vs. enterprise unit & - & -0.300 & 1.348 & 0.316 & -0.349 & 1.930 & - & - & 1.454 \\
\hline Farmer vs. enterprise unit & -0.473 & - & - & -0.898 & -0.561 & -0.333 & -2.097 & -0.584 & -0.574 \\
\hline Self-employed vs. enterprise unit & - & - & 0.686 & -1.157 & -0.384 & 0.558 & -2.540 & -1.114 & - \\
\hline Others vs. enterprise unit & -0.844 & -0.193 & 0.679 & -1.480 & -0.475 & 0.616 & -2.160 & -0.704 & 0.473 \\
\hline Monthly income & - & -0.273 & - & - & -0.221 & - & -0.163 & -0.222 & - \\
\hline \multicolumn{10}{|l|}{ Travel purpose } \\
\hline Mandatory travel vs. leisure travel & -0.477 & -0.284 & -0.425 & -0.341 & -0.388 & - & - & -0.230 & 0.138 \\
\hline Intercity travel distance & 0.004 & 0.001 & - & 0.002 & - & -0.002 & 0.003 & - & -0.002 \\
\hline Intercity travel cost & 0.018 & 0.015 & - & 0.027 & 0.022 & 0.001 & 0.026 & 0.023 & - \\
\hline Intercity travel time & -1.239 & -0.397 & - & -1.265 & -0.417 & 0.002 & -1.255 & -0.482 & 0.036 \\
\hline Safety & 0.566 & 0.609 & - & 0.818 & 0.685 & - & -0.098 & 0.621 & - \\
\hline Comfort & - & 0.314 & -0.433 & 0.195 & 0.369 & -0.463 & 0.639 & 0.312 & -0.480 \\
\hline Punctuality & -0.335 & 0.319 & -0.574 & -0.377 & 0.317 & -0.525 & -0.496 & 0.323 & -0.817 \\
\hline Access time & 0.390 & - & -0.268 & 0.455 & - & -0.284 & 0.351 & -0.220 & - \\
\hline Departure time & 0.694 & - & - & 1.026 & 0.264 & - & 0.796 & - & -0.427 \\
\hline Constant & -7.703 & -5.803 & 3.089 & -9.503 & -6.798 & 2.328 & -5.985 & -5.589 & 3.884 \\
\hline
\end{tabular}

Note: parameters that were significant at the $95 \%$ confidence level are shown in the table.

For the predictive performance of the models, we found that MLP performs best regardless of oversampling or undersampling balanced data. More importantly, BMNL and MNL show the same predictive performance when using oversampling balanced data, and RBF models have the worst predictive performance. Similarly, BMNL and MNL have the same predictive performance using undersampled data, but their performance is lower than that of RBF.

The fitting performance of models based on balanced data is a slight improvement over using imbalanced data. For example, the fitting performance of MLP model is $80.70 \%$ using imbalanced data, and $81.80 \%$ and $83.10 \%$, respectively, with undersampled and oversampled data. However, except for the RBF model, the predictive performance of these models based on balanced data is slightly lower than that when using imbalanced data.

The ROC curve was also used to intuitively judge the predictive performance of each model, and AUCs were used to quantitatively compare their predictive accuracy under different modeling techniques. We found that the results 
TABLE 5: Parameter estimation in BMNL using imbalanced data.

\begin{tabular}{|c|c|c|c|c|c|c|c|c|c|c|c|c|}
\hline \multirow[b]{2}{*}{ Variable } & \multicolumn{4}{|c|}{ Airplane } & \multicolumn{4}{|c|}{ HSR } & \multicolumn{4}{|c|}{ Express bus } \\
\hline & Mean & SD & $\begin{array}{l}\text { Credit } \\
2.50 \%\end{array}$ & $\begin{array}{l}\text { interval } \\
97.50 \%\end{array}$ & Mean & SD & $\begin{array}{l}\text { Credit } \\
2.50 \%\end{array}$ & $\begin{array}{l}\text { interval } \\
97.50 \%\end{array}$ & Mean & SD & $\begin{array}{l}\text { Credit i } \\
2.50 \%\end{array}$ & $\begin{array}{r}\text { interval } \\
97.50 \%\end{array}$ \\
\hline \multicolumn{13}{|l|}{ Gender } \\
\hline Male vs. & 0.555 & 0.143 & 0.275 & 0.833 & 0.186 & 0.057 & 0.067 & 0.295 & 0.368 & 0.167 & 0.040 & 0.677 \\
\hline Age & 0.282 & 0.089 & 0.114 & 454 & 0.350 & 0.091 & 0.180 & 0.538 & 0.247 & 0.084 & .072 & 0.407 \\
\hline \multicolumn{13}{|l|}{ Occupation } \\
\hline $\begin{array}{l}\text { Personnel of institution vs. } \\
\text { enterprise unit }\end{array}$ & 0.710 & 0.173 & 0.377 & 1.051 & 0.408 & 0.059 & 0.295 & 0.527 & 0.651 & 0.129 & 0.403 & 0.913 \\
\hline Student vs. enterprise unit & 0.114 & 0.171 & -0.217 & 0.435 & -0.300 & 0.068 & -0.440 & -0.167 & 1.348 & 0.181 & 1.002 & 1.727 \\
\hline Farmer vs. enterprise unit & -0.473 & 0.226 & -0.930 & -0.027 & -0.078 & 0.063 & -0.205 & 0.423 & -0.031 & 0.158 & -0.321 & 0.288 \\
\hline Self-employed vs. enterprise unit & -0.147 & 0.187 & -0.522 & 0.228 & 0.074 & 0.082 & -0.083 & 0.235 & 0.686 & 0.139 & 0.409 & 0.967 \\
\hline Others vs. enterprise unit & -0.844 & 0.161 & -1.173 & -0.526 & -0.193 & 0.066 & -0.316 & -0.059 & 0.679 & 0.144 & 0.396 & 0.953 \\
\hline Monthly income & -0.098 & 0.090 & -0.270 & 0.089 & -0.273 & 0.062 & -0.394 & -0.154 & -0.121 & 0.070 & -0.251 & 0.016 \\
\hline \multicolumn{13}{|l|}{ Travel purpose } \\
\hline Mandatory travel vs. le & -0.477 & 0.196 & -0.871 & -0.096 & -0.284 & 0.053 & -0.384 & -0.185 & -0.425 & 0.130 & -0.699 & -0.192 \\
\hline Intercity travel distance & 0.004 & 0.001 & 0.003 & 0.005 & 0.001 & 0.001 & 0.001 & 0.002 & -0.001 & 0.001 & -0.002 & 0.001 \\
\hline Intercity travel cost & 0.018 & 0.002 & 0.014 & 0.022 & 0.015 & 0.002 & 0.011 & 0.186 & 0.001 & 0.002 & -0.005 & 0.004 \\
\hline Intercity travel time & -1.239 & 0.096 & -1.433 & -1.059 & -0.397 & 0.044 & -0.485 & -0.311 & -0.009 & 0.029 & -0.064 & 0.048 \\
\hline Safety & 0.566 & 0.149 & 0.268 & 0.847 & 0.609 & 0.086 & 0.440 & 0.782 & 0.039 & 0.065 & -0.079 & 0.173 \\
\hline Comfort & 0.245 & 0.124 & -0.017 & 0.474 & 0.314 & 0.053 & 0.211 & 0.427 & -0.433 & 0.099 & -0.634 & -0.254 \\
\hline Punctuality & -0.335 & 0.13 & -0.586 & -0.089 & 0.319 & 0.066 & 0.189 & 0.440 & -0.574 & 0.04 & -0.655 & -0.498 \\
\hline Access time & 0.390 & 0.151 & 0.074 & 0.677 & -0.08 & 0.056 & -0.189 & 0.034 & -0.268 & 0.106 & -0.472 & -0.066 \\
\hline Departure time & 0.694 & 0.142 & 0.428 & 0.981 & 0.029 & 0.060 & -0.080 & 0.151 & -0.161 & 0.132 & -0.419 & 0.094 \\
\hline Constant & -7.703 & 0.081 & -7.863 & -7.537 & -5.803 & 0.098 & -5.997 & -5.62 & 3.089 & 0.124 & 2.857 & 3.342 \\
\hline
\end{tabular}

TABLE 6: Parameter estimation in BMNL using oversampling of balanced data.

\begin{tabular}{|c|c|c|c|c|c|c|c|c|c|c|c|c|}
\hline \multirow[b]{2}{*}{ Variable } & \multicolumn{4}{|c|}{ Airplane } & \multicolumn{4}{|c|}{ HSR } & \multicolumn{4}{|c|}{ Express bus } \\
\hline & Mean & SD & $\begin{array}{l}\text { Credit } \\
2.50 \% \\
\end{array}$ & $\begin{array}{r}\text { interval } \\
97.50 \% \\
\end{array}$ & Mean & SD & $\begin{array}{l}\text { Credit } \\
2.50 \% \\
\end{array}$ & $\begin{array}{r}\text { interval } \\
97.50 \%\end{array}$ & Mean & SD & $\begin{array}{l}\text { Credit } \\
2.50 \% \\
\end{array}$ & $\begin{array}{r}\text { interval } \\
97.50 \% \\
\end{array}$ \\
\hline \multicolumn{13}{|l|}{ Gender } \\
\hline Male vs. $\mathrm{f}$ & 0.683 & 0.118 & 0.474 & 0.947 & 0.197 & 0.075 & 0.052 & 0.339 & 0.713 & 0.085 & 0.556 & 0.872 \\
\hline Age & 0.416 & 0.106 & 0.190 & 0.615 & 0.357 & 0.028 & 0.298 & 0.410 & 0.346 & 0.054 & 0.241 & 0.452 \\
\hline \multicolumn{13}{|l|}{ Occupation } \\
\hline $\begin{array}{l}\text { Personnel of institution vs. } \\
\text { enterprise unit }\end{array}$ & 0.344 & 0.076 & 0.218 & 0.510 & 0.143 & 0.054 & 0.041 & 0.256 & 0.313 & 0.107 & 0.050 & 0.491 \\
\hline Student vs. enterprise unit & 0.316 & 0.078 & 0.126 & 0.436 & -0.349 & 0.050 & -0.444 & -0.256 & 1.930 & 0.060 & 1.817 & 2.041 \\
\hline Farmer vs. enterprise unit & -0.898 & 0.058 & -1.014 & -0.787 & -0.561 & 0.077 & -0.712 & -0.417 & -0.333 & 0.054 & -0.438 & -0.229 \\
\hline Self-employed vs. enterprise unit & -1.157 & 0.120 & -1.398 & -0.928 & -0.384 & 0.075 & -0.531 & -0.232 & 0.558 & 0.142 & 0.269 & 0.818 \\
\hline Other & 1.480 & 0.072 & -1.643 & -1.348 & -0.475 & 0.116 & -0.695 & & & 0.101 & & 0.794 \\
\hline Monthly income & 0.044 & 0.061 & -0.192 & 0.058 & -0.221 & 0.047 & -0.315 & -0.129 & 0.015 & 0.052 & -0.079 & 0.128 \\
\hline \multicolumn{13}{|c|}{ 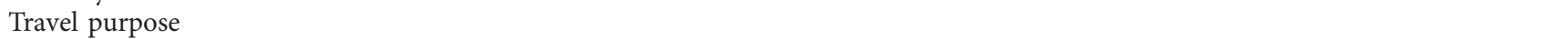 } \\
\hline Mandatory tr & -0.341 & 0.033 & -0.396 & -0.264 & -0.388 & 0.086 & -0 . & -0.216 & -0.127 & 0.064 & -0 & 0.004 \\
\hline Interci & 0.002 & 0.001 & & & -0.001 & 0.001 & & 0.001 & -0.002 & 0.001 & -0 & -0.001 \\
\hline Intercity travel cost & 0.027 & 0.002 & 0.023 & 0.031 & 0.022 & 0.002 & 0.018 & 0.026 & 0.001 & 0.002 & -0.002 & 0.005 \\
\hline Intercity travel time & -1.265 & 0.075 & -1.426 & -1.128 & -0.417 & 0.022 & -0.459 & -0.373 & 0.002 & 0.024 & -0.047 & 0.045 \\
\hline Safety & 0.818 & 0.069 & 0.671 & 0.947 & 0.685 & 0.047 & 0.594 & 0.780 & 0.155 & 0.104 & -0.042 & 0.359 \\
\hline Comfort & 0.195 & 0.120 & 0.007 & 0.490 & 0.369 & 0.033 & 0.303 & 0.434 & -0.463 & 0.094 & -0.644 & -0.284 \\
\hline Punctuality & -0.377 & 0.116 & -0.602 & -0.164 & 0.317 & 0.041 & 0.242 & 0.399 & -0.525 & 0.080 & -0.680 & -0.370 \\
\hline Access time & 0.455 & 0.081 & 0.255 & 0.587 & -0.136 & 0.110 & -0.357 & 0.066 & -0.284 & 0.058 & -0.409 & -0.175 \\
\hline Departure time & 1.026 & 0.171 & 0.717 & 1.356 & 0.264 & 0.033 & 0.201 & 0.329 & -0.202 & 0.111 & -0.418 & 0.012 \\
\hline Constant & -9.503 & 0.080 & -9.678 & -9.362 & -6.798 & 0.092 & -6.976 & -6.605 & 2.328 & 0.067 & 2.164 & 2.433 \\
\hline
\end{tabular}


TABLe 7: Parameter estimation in BMNL using undersampling of balanced data.

\begin{tabular}{|c|c|c|c|c|c|c|c|c|c|c|c|c|}
\hline \multirow[b]{2}{*}{ Variable } & \multicolumn{4}{|c|}{ Airplane } & \multicolumn{4}{|c|}{ HSR } & \multicolumn{4}{|c|}{ Express bus } \\
\hline & Mean & SD & $\begin{array}{l}\text { Credit } \\
2.50 \% \\
\end{array}$ & $\begin{array}{r}\text { interval } \\
97.50 \% \\
\end{array}$ & Mean & SD & $\begin{array}{l}\text { Credit } \\
2.50 \% \\
\end{array}$ & $\begin{array}{r}\text { interval } \\
97.50 \% \\
\end{array}$ & Mean & SD & $\begin{array}{l}\text { Credit } \\
2.50 \% \\
\end{array}$ & $\begin{array}{r}\text { interval } \\
97.50 \% \\
\end{array}$ \\
\hline \multicolumn{13}{|l|}{ Gender } \\
\hline Male vs. $\mathrm{f}$ & 1.231 & 0.266 & 0.719 & 1.764 & 0.384 & 0.114 & 0.167 & 0.620 & 0.733 & 0.165 & 0.435 & 1.075 \\
\hline Age & 0.396 & 0.102 & 0.206 & 0.595 & 0.232 & 0.143 & -0.080 & 0.506 & 0.380 & 0.083 & 0.220 & 0.541 \\
\hline \multicolumn{13}{|l|}{ Occupation } \\
\hline $\begin{array}{l}\text { Personnel of institution vs. } \\
\text { enterprise unit }\end{array}$ & -0.162 & 0.130 & -0.403 & 0.081 & 0.176 & 0.106 & -0.393 & 035 & 0.139 & 0.171 & -0.235 & 0.444 \\
\hline Student vs. enterprise unit & 0.041 & 0.170 & -0.274 & 0.366 & -0.121 & 0.210 & -0.529 & 0.288 & 1.454 & 0.131 & 1.198 & 1.718 \\
\hline Farmer vs. enterprise unit & -2.097 & 0.191 & -2.450 & -1.722 & -0.584 & 0.061 & -0.709 & -0.458 & -0.574 & 0.223 & -1.017 & -0.128 \\
\hline Self-employed vs. enterprise unit & -2.540 & 0.232 & -3.011 & -2.105 & -1.114 & 0.081 & -1.280 & -0.954 & 0.021 & 0.231 & -0.449 & \\
\hline Others & & 0.211 & & & & 0.085 & -0.906 & & & 0.134 & 0.217 & 0.740 \\
\hline Monthly income & -0.163 & 0.067 & -0.305 & -0.031 & -0.222 & 0.101 & -0.418 & -0.027 & -0.128 & 0.075 & -0.284 & 0.020 \\
\hline \multicolumn{13}{|l|}{ Travel purpose } \\
\hline Mandatory trav & -0.038 & 0.215 & -0.470 & & -0.230 & 0.113 & -0.471 & -0.013 & & 0.205 & & 0.5 \\
\hline Intercity travel distance & 0.003 & 0.001 & & 0.005 & 0.000 & 0.001 & -0.002 & 0.002 & -0.002 & 0.001 & -0.004 & -0.001 \\
\hline Intercity travel cost & 0.026 & 0.004 & 0.020 & 0.035 & 0.023 & 0.003 & 0.016 & 0.031 & 0.000 & 0.003 & -0.006 & 0.006 \\
\hline Intercity travel time & -1.255 & 0.062 & -1.378 & -1.146 & -0.482 & 0.056 & -0.598 & -0.388 & 0.036 & 0.043 & -0.049 & 0.126 \\
\hline Safety & -0.098 & 0.133 & -0.357 & 0.151 & 0.621 & 0.099 & 0.432 & 0.824 & 0.077 & 0.096 & -0.113 & 0.274 \\
\hline Comfort & 0.639 & 0.035 & 0.574 & 0.706 & 0.312 & 0.120 & 0.056 & 0.526 & -0.480 & 0.134 & -0.753 & -0.223 \\
\hline Punctuality & -0.496 & 0.158 & -0.800 & -0.190 & 0.323 & 0.018 & 0.290 & 0.363 & -0.817 & 0.118 & -1.065 & -0.582 \\
\hline Access time & 0.351 & 0.073 & 0.216 & 0.490 & -0.220 & 0.030 & -0.278 & -0.159 & 0.042 & 0.070 & -0.098 & 0.178 \\
\hline Departure time & 0.796 & 0.109 & 0.584 & 1.029 & -0.050 & 0.063 & -0.178 & 0.064 & -0.427 & 0.115 & -0.641 & -0.200 \\
\hline Constant & -5.985 & 0.135 & -6.242 & -5.731 & -5.589 & 0.207 & -6.001 & -5.200 & 3.884 & 0.173 & 3.540 & 4.203 \\
\hline
\end{tabular}

TABLE 8: Parameter estimation in MNL.

\begin{tabular}{|c|c|c|c|c|c|c|c|c|c|}
\hline \multirow{3}{*}{ Variable } & \multicolumn{3}{|c|}{ Imbalanced data } & \multicolumn{3}{|c|}{ Oversampling balanced data } & \multicolumn{3}{|c|}{ Undersampling balanced data } \\
\hline & Airplane & HSR & $\begin{array}{l}\text { Express } \\
\text { bus }\end{array}$ & Airplane & HSR & $\begin{array}{c}\text { Express } \\
\text { bus }\end{array}$ & Airplane & HSR & $\begin{array}{l}\text { Express } \\
\text { bus }\end{array}$ \\
\hline & Coefficient & Coefficient & Coefficient & Coefficient & Coefficient & Coefficient & Coefficient & Coefficient & Coefficient \\
\hline \multicolumn{10}{|l|}{ Gender } \\
\hline Male vs. female & - & - & - & - & - & 0.681 & - & - & 0.723 \\
\hline Age & - & 0.349 & - & - & 0.366 & 0.360 & - & - & - \\
\hline \multicolumn{10}{|l|}{ Occupation } \\
\hline $\begin{array}{l}\text { Personnel of } \\
\text { institutions vs. } \\
\text { enterprise unit }\end{array}$ & - & - & - & - & - & - & - & - & - \\
\hline $\begin{array}{l}\text { Student vs. } \\
\text { enterprise unit }\end{array}$ & - & - & 1.298 & - & -0.352 & 1.856 & - & - & 1.642 \\
\hline $\begin{array}{l}\text { Farmer vs. } \\
\text { enterprise unit }\end{array}$ & - & - & - & - & - & - & - & - & - \\
\hline $\begin{array}{l}\text { Self-employed vs. } \\
\text { enterprise unit }\end{array}$ & - & - & - & - & - & - & - & - & - \\
\hline $\begin{array}{l}\text { Others vs. enterprise } \\
\text { unit }\end{array}$ & - & - & - & - & - & - & - & - & - \\
\hline Monthly income & -0.128 & -0.289 & - & - & -0.226 & - & - & - & - \\
\hline \multicolumn{10}{|l|}{ Travel purpose } \\
\hline $\begin{array}{l}\text { Mandatory travel vs. } \\
\text { leisure travel }\end{array}$ & - & - & - & - & - & - & - & - & - \\
\hline $\begin{array}{l}\text { Intercity travel } \\
\text { distance }\end{array}$ & 0.004 & 0.001 & - & 0.002 & - & -0.002 & 0.003 & - & - \\
\hline Intercity travel cost & 0.017 & 0.014 & - & 0.026 & 0.022 & - & 0.031 & 0.027 & - \\
\hline Intercity travel time & -1.211 & -0.380 & - & -1.242 & -0.421 & - & -1.395 & -0.638 & - \\
\hline Safety & - & 0.585 & - & 0.801 & 0.678 & - & - & - & - \\
\hline Comfort & - & - & -0.458 & - & - & -0.461 & - & - & -0.418 \\
\hline Punctuality & - & 0.299 & -0.575 & - & - & -0.513 & - & - & -0.617 \\
\hline Access time & - & - & - & - & - & - & - & - & - \\
\hline Departure time & 0.731 & - & - & 1.030 & - & -0.185 & - & - & - \\
\hline Constant & -7.673 & -5.821 & 3.058 & -9.522 & -6.798 & 2.303 & - & -5.297 & 3.420 \\
\hline
\end{tabular}

Note: parameters that were significant at the $95 \%$ confidence level are shown in the table. 
TABLE 9: Parameter estimation in MNL using imbalanced data.

\begin{tabular}{|c|c|c|c|c|c|c|c|c|c|c|c|c|}
\hline \multirow{3}{*}{ Variable } & \multicolumn{4}{|c|}{ Airplane } & \multicolumn{4}{|c|}{ HSR } & \multicolumn{4}{|c|}{ Express bus } \\
\hline & \multirow{2}{*}{ Coefficient } & \multirow{2}{*}{$P>z$} & \multicolumn{2}{|c|}{ Credit interval } & \multirow{2}{*}{ Coefficient } & \multirow{2}{*}{$P>z$} & \multicolumn{2}{|c|}{ Credit interval } & \multirow{2}{*}{ Coefficient } & \multirow{2}{*}{$P>z$} & \multicolumn{2}{|c|}{ Credit interval } \\
\hline & & & $2.50 \%$ & $97.50 \%$ & & & $2.50 \%$ & $97.50 \%$ & & & $2.50 \%$ & $97.50 \%$ \\
\hline \multicolumn{13}{|l|}{ Gender } \\
\hline Male vs. female & 0.474 & 0.321 & -0.462 & 1.410 & 0.160 & 0.543 & -0.355 & 0.674 & 0.409 & 0.121 & -0.107 & 0.926 \\
\hline Age & 0.326 & 0.269 & -0.252 & 0.904 & 0.349 & 0.035 & 0.024 & 0.675 & & 0.128 & -0.073 & 0.579 \\
\hline \multicolumn{13}{|l|}{ Occupation } \\
\hline $\begin{array}{l}\text { Personnel of } \\
\text { institution vs. } \\
\text { enterprise unit }\end{array}$ & 0.725 & 0.290 & -0.618 & 2.069 & 0.410 & 0.316 & -0.391 & 1.210 & 42 & 0.191 & -0.320 & 1.604 \\
\hline $\begin{array}{l}\text { Student vs. enterprise } \\
\text { unit }\end{array}$ & 0.116 & 0.885 & -1.453 & 1.685 & -0.362 & 0.390 & -1.189 & 0.464 & 1.298 & 0.002 & 0.464 & 2.132 \\
\hline $\begin{array}{l}\text { Farmer vs. enterprise } \\
\text { unit }\end{array}$ & -0.425 & 0.658 & -2 & 1.458 & -0.080 & 0.892 & -1.234 & 1.074 & & 0.929 & -1.359 & 1.241 \\
\hline $\begin{array}{l}\text { Self-employed vs. } \\
\text { enterprise unit }\end{array}$ & -0.207 & 0.789 & -1.720 & 1.306 & 0.081 & 0.849 & -0.754 & 0.916 & 0.611 & 0.195 & -0.314 & 1.537 \\
\hline $\begin{array}{l}\text { Others vs. enterprise } \\
\text { unit }\end{array}$ & -0.824 & 0.366 & -2.610 & 0.962 & -0.173 & 0.732 & -1.161 & 0.816 & 0.705 & 0.178 & -0.32 & 1.730 \\
\hline Monthly income & -0.128 & 0.565 & -0.563 & 0.307 & -0.289 & 0.013 & -0.516 & -0.062 & -0.106 & 0.329 & -0.318 & 0.106 \\
\hline \multicolumn{13}{|l|}{ Travel purpose } \\
\hline $\begin{array}{l}\text { Mandatory travel vs. } \\
\text { leisure travel }\end{array}$ & -0.443 & 0.340 & -1.352 & 0.466 & -0.302 & 0.256 & -0.823 & 0.219 & -0.412 & 0.112 & -0.921 & 0.096 \\
\hline Intercity travel distance & 0.004 & 0.001 & 0.002 & 0.005 & 0.001 & 0.278 & -0.001 & 0.002 & -0.001 & 0.009 & -0.003 & 0.001 \\
\hline Intercity travel cost & 0.017 & 0.001 & 0.013 & 0.021 & 0.014 & 0.001 & 0.010 & 0.018 & 0.001 & 0.828 & -0.005 & 0.004 \\
\hline Intercity travel time & -1.211 & 0.001 & -1.444 & -0.978 & -0.380 & 0.001 & -0.483 & -0.276 & -0.003 & 0.917 & -0.061 & 0.055 \\
\hline Safety & 0.491 & 0.137 & -0.156 & 1.138 & 0.585 & 0.004 & 0.188 & 0.982 & 0.059 & 0.760 & -0.319 & 0.437 \\
\hline Comfort & 0.251 & 0.410 & -0.345 & 0.846 & 0.358 & 0.056 & -0.01 & 0.725 & -0.458 & 0.011 & -0.811 & -0.105 \\
\hline Punctuality & -0.278 & 0.356 & -0.866 & 0.311 & 0.299 & 0.104 & -0.061 & 0.660 & -0.575 & 0.002 & -0.936 & -0.213 \\
\hline Access time & 0.404 & 0.180 & -0.187 & 0.994 & -0.035 & 0.841 & -0.374 & 0.304 & -0.221 & 0.172 & -0.539 & 0.096 \\
\hline Departure time & 0.731 & 0.017 & 0.131 & 1.331 & 0.047 & 0.791 & -0.304 & 0.398 & -0.210 & 0.269 & -0.584 & 0.163 \\
\hline Constant & -7.673 & 0.002 & -12.518 & -2.828 & -5.821 & 0.001 & -8.592 & -3.051 & 3.058 & 0.012 & 0.667 & 5.450 \\
\hline
\end{tabular}

TABLE 10: Parameter estimation in MNL using oversampling of balanced data.

\begin{tabular}{|c|c|c|c|c|c|c|c|c|c|c|c|c|}
\hline \multirow{3}{*}{ Variable } & \multicolumn{4}{|c|}{ Airplane } & \multicolumn{4}{|c|}{ HSR } & \multicolumn{4}{|c|}{ Express bus } \\
\hline & \multirow{2}{*}{ Coefficient } & \multirow{2}{*}{$P>z$} & \multicolumn{2}{|c|}{ Credit interval } & \multirow{2}{*}{ Coefficient } & \multirow{2}{*}{$P>z$} & \multicolumn{2}{|c|}{ Credit interval } & \multirow{2}{*}{ Coefficient } & \multirow{2}{*}{$P>z$} & \multicolumn{2}{|c|}{ Credit interval } \\
\hline & & & $2.50 \%$ & $97.50 \%$ & & & $2.50 \%$ & $97.50 \%$ & & & $2.50 \%$ & $97.50 \%$ \\
\hline \multicolumn{13}{|l|}{ Gender } \\
\hline Male vs. f & 0.661 & 0.118 & -0.167 & 1.490 & 0.196 & 0.443 & -0.305 & 0.697 & 0.681 & 0.001 & 0.275 & 1.087 \\
\hline Age & 6 & 0.126 & -0.108 & 0.880 & 6 & 0.020 & 0.058 & 0.673 & 50 & 0.007 & 0.097 & 0.624 \\
\hline \multicolumn{13}{|l|}{ Occupation } \\
\hline $\begin{array}{l}\text { Personnel of } \\
\text { institution vs. } \\
\text { enterprise unit }\end{array}$ & 0.343 & 0.564 & -0.824 & 1.510 & 0.138 & 0.723 & -0.627 & 0.903 & 0.318 & 0.401 & -0.424 & 1.061 \\
\hline $\begin{array}{l}\text { Student vs. enterprise } \\
\text { unit }\end{array}$ & 0.298 & 0.672 & -1.082 & 1.679 & -0.352 & 0.399 & -1.171 & 0.466 & 1.856 & 0.001 & 1.193 & 2.519 \\
\hline $\begin{array}{l}\text { Farmer vs. enterprise } \\
\text { unit }\end{array}$ & -0.898 & 0.309 & -2.627 & 0.831 & -0.546 & 0.376 & -1.757 & 0.664 & -0.338 & 0.488 & -1.291 & 0.616 \\
\hline $\begin{array}{l}\text { Self-employed vs. } \\
\text { enterprise unit }\end{array}$ & -1.107 & 0.126 & -2.525 & 0.312 & -0.387 & 0.376 & -1.243 & 0.469 & 0.554 & 0.119 & -0.142 & 1.250 \\
\hline $\begin{array}{l}\text { Others vs. enterprise } \\
\text { unit }\end{array}$ & -1.518 & 0.055 & -3.067 & 0.030 & -0.479 & 0.305 & -1.394 & 0.437 & 0.545 & 0.179 & -0.250 & 1.340 \\
\hline Monthly income & -0.040 & 0.836 & -0.416 & 0.336 & -0.226 & 0.043 & -0.445 & -0.007 & -0.017 & 0.840 & -0.182 & 0.148 \\
\hline \multicolumn{13}{|l|}{ Travel purpose } \\
\hline $\begin{array}{l}\text { Mandatory travel vs. } \\
\text { leisure travel }\end{array}$ & -0.343 & 0.413 & -1.166 & 0.479 & -0.384 & 0.140 & -0.895 & 0.126 & -0.134 & 0.506 & -0.529 & 0.261 \\
\hline Intercity travel distance & 0.002 & 0.001 & 0.001 & 0.004 & -0.001 & 0.264 & -0.002 & 0.001 & -0.002 & 0.001 & -0.003 & -0.001 \\
\hline Intercity travel cost & 0.026 & 0.001 & 0.021 & 0.031 & 0.022 & 0.001 & 0.017 & 0.026 & 0.001 & 0.580 & -0.003 & 0.005 \\
\hline
\end{tabular}


Table 10: Continued.

\begin{tabular}{|c|c|c|c|c|c|c|c|c|c|c|c|c|}
\hline \multirow{3}{*}{ Variable } & \multicolumn{4}{|c|}{ Airplane } & \multicolumn{4}{|c|}{ HSR } & \multicolumn{4}{|c|}{ Express bus } \\
\hline & \multirow{2}{*}{ Coefficient } & \multirow{2}{*}{$P>z$} & \multicolumn{2}{|c|}{ Credit interval } & \multirow{2}{*}{ Coefficient } & \multirow{2}{*}{$P>z$} & \multicolumn{2}{|c|}{ Credit interval } & \multirow{2}{*}{ Coefficient } & \multirow{2}{*}{$P>z$} & \multicolumn{2}{|c|}{ Credit interval } \\
\hline & & & $2.50 \%$ & $97.50 \%$ & & & $2.50 \%$ & $97.50 \%$ & & & $2.50 \%$ & $97.50 \%$ \\
\hline Intercity travel time & -1.242 & 0.001 & -1.432 & -1.053 & -0.421 & 0.001 & -0.524 & -0.318 & 0.001 & 0.964 & -0.050 & 0.052 \\
\hline Safety & 0.801 & 0.006 & 0.225 & 1.378 & 0.678 & 0.001 & 0.279 & 1.077 & 0.146 & 0.333 & -0.149 & 0.440 \\
\hline Comfort & 0.236 & 0.404 & -0.318 & 0.791 & 0.371 & 0.051 & -0.001 & 0.743 & -0.461 & 0.001 & -0.737 & -0.185 \\
\hline Punctuality & -0.353 & 0.194 & -0.886 & 0.179 & 0.321 & 0.082 & -0.040 & 0.682 & -0.513 & 0.001 & -0.808 & -0.219 \\
\hline Access time & 0.456 & 0.076 & -0.048 & 0.959 & -0.122 & 0.462 & -0.447 & 0.203 & -0.244 & 0.060 & -0.499 & 0.010 \\
\hline Departure time & 1.030 & 0.001 & 0.506 & 1.554 & 0.271 & 0.130 & -0.080 & 0.621 & -0.185 & 0.232 & -0.487 & 0.118 \\
\hline Constant & -9.522 & 0.001 & -13.873 & -5.170 & -6.798 & 0.001 & -9.530 & -4.066 & 2.303 & 0.020 & 0.356 & 4.250 \\
\hline
\end{tabular}

TABLE 11: Parameter estimation in MNL using undersampling of balanced data.

\begin{tabular}{|c|c|c|c|c|c|c|c|c|c|c|c|c|}
\hline \multirow[b]{2}{*}{ Variable } & \multicolumn{4}{|c|}{ Airplane } & \multicolumn{4}{|c|}{ HSR } & \multicolumn{4}{|c|}{ Express bus } \\
\hline & Coefficient & $P>z$ & \multicolumn{2}{|c|}{ Credit interval } & Coefficient & $P>z$ & \multicolumn{2}{|c|}{ Credit interval } & Coefficient & $P>z$ & \multicolumn{2}{|c|}{ Credit interval } \\
\hline \multicolumn{13}{|l|}{ Gender } \\
\hline Male vs. female & 0.847 & 0.137 & -0.268 & 1.962 & 0.175 & 0.637 & -0.552 & 0.901 & 0.723 & 0.010 & 0.176 & 1.271 \\
\hline Age & 0.439 & 0.213 & -0.252 & 1.130 & 0305 & 0.183 & -0.144 & 0.754 & & 0.135 & -0.080 & 0.598 \\
\hline \multicolumn{13}{|l|}{ Occupation } \\
\hline $\begin{array}{l}\text { Personnel of } \\
\text { institution vs. } \\
\text { enterprise unit }\end{array}$ & 0.252 & 0.748 & -1.284 & 1.787 & -0.057 & 0.916 & -1.113 & 0.999 & 0.220 & 0.657 & -0.751 & 1.191 \\
\hline $\begin{array}{l}\text { Student vs. enterprise } \\
\text { unit }\end{array}$ & 0.317 & 0.742 & -1.565 & 2.199 & & 0.831 & -1.304 & 1.048 & 1.642 & 0.001 & 0.774 & 2.510 \\
\hline $\begin{array}{l}\text { Farmer vs. enterprise } \\
\text { unit }\end{array}$ & -1.600 & 0.179 & -3 & 0732 & -0.7 & 0.399 & -2.339 & 0.931 & -0 & 0.420 & -1.777 & 0.741 \\
\hline $\begin{array}{l}\text { Self-employed vs. } \\
\text { enterprise unit }\end{array}$ & -1.939 & 0.059 & -3.953 & 0.076 & -0.828 & 0.200 & -2.096 & 0.439 & 0.646 & 0.176 & -0.291 & 1.584 \\
\hline $\begin{array}{l}\text { Others vs. enterprise } \\
\text { unit }\end{array}$ & -1.860 & 0.093 & -4.028 & 0.307 & 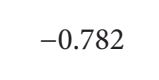 & 0.289 & -2.229 & 0.664 & 0.632 & 0.256 & -0.458 & 1.721 \\
\hline Monthly income & -0.139 & 0.612 & -0.676 & 0.398 & -0223 & 0.180 & -0.550 & 0.103 & -0.048 & 0.671 & -0.271 & 0.175 \\
\hline \multicolumn{13}{|l|}{ Travel purpose } \\
\hline $\begin{array}{l}\text { Mandatory travel vs. } \\
\text { leisure travel }\end{array}$ & -0.337 & 0.549 & -1.439 & 0.764 & -0.313 & 0.408 & -1.055 & 0.428 & -0.283 & 0.300 & -0.819 & 0.252 \\
\hline Intercity travel distance & 0 & 0.006 & 0.001 & $0 .($ & 0.0 & 0.960 & -0.002 & 0.0 & -0.002 & 0.008 & -0.003 & 0.001 \\
\hline Intercity travel cost & & 0.001 & & 0.0 & & 0.001 & 0.019 & & 0.0 & 0.875 & -0.006 & 0.005 \\
\hline Intercity travel time & -1.395 & 0.001 & -1.686 & -1.105 & -0.638 & 0.001 & -0.837 & -0.439 & -0.013 & 0.763 & -0.095 & 0.070 \\
\hline Safety & 0.084 & 0.838 & -0.727 & 0.896 & 0.514 & 0.074 & -0.050 & 1.077 & 0.014 & 0.945 & -0.392 & 0.421 \\
\hline Comfort & 0.301 & 0.424 & -0.437 & 1.039 & 0.466 & 0.074 & -0.044 & 0.976 & -0.418 & 0.023 & -0.780 & -0.057 \\
\hline Punctuality & -0.389 & 0.256 & -1.061 & 0.283 & 0.189 & 0.448 & -0.298 & 0.675 & -0.617 & 0.002 & -1.003 & -0.230 \\
\hline Access time & 0.422 & 0.237 & -0.277 & 1.120 & -0.194 & 0.430 & -0.676 & 0.288 & -0.135 & 0.434 & -0.475 & 0.204 \\
\hline Departure time & 0.655 & 0.073 & -0.061 & 1.371 & -0.034 & 0.892 & -0.530 & 0.461 & -0.226 & 0.259 & -0.617 & 0.166 \\
\hline Constant & -5.696 & 0.050 & -11.396 & 0.004 & -5.297 & 0.006 & -9.039 & -1.555 & 3.420 & 0.008 & 0.912 & 5.928 \\
\hline
\end{tabular}

from AUCs are consistent with those from the confusion matrices for each model.

4.3. Model Interpretation. We use the results of the BMNL with better statistical performance and MLP models with better predictive performance to explain the effects of factors on intercity travel mode choice.

From Table 4 and Figure 4, it is found that gender was positively correlated with the choice of HSR and express bus, indicating that men were prone to traveling by HSR or express bus, and women by train. This finding is consistent with a previous study [2], which revealed that women preferred using train more than men. The models show that personnel of government-sponsored institutions were more likely than enterprise personnel to choose an airplane. Farmers and the self-employed were less likely than enterprise personnel to travel by airplane. Similarly, students and farmers were not prone to choosing HSR, and farmers were prone to using an express bus $[2,8]$. These results are supported by a previous study $[1,3]$ that found that passengers working in the state sector are likely to choose airplane over coach. Monthly income was found to be positively associated with airplane choice, and negatively 


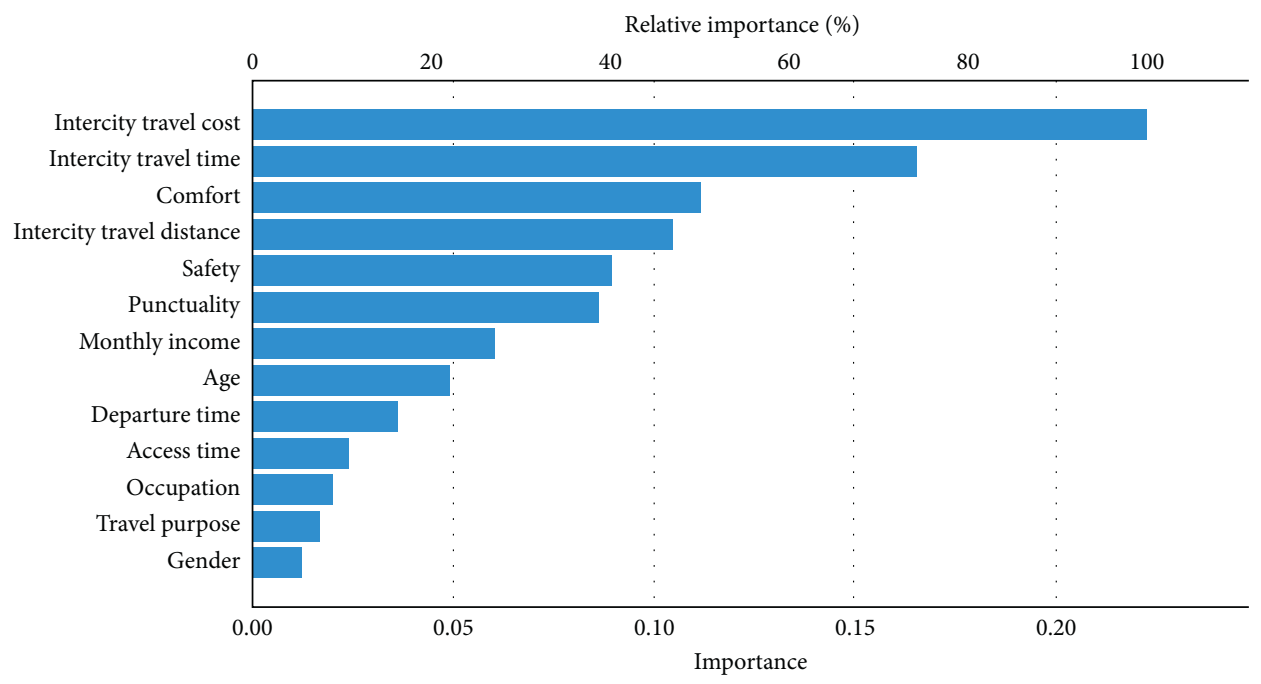

(a)

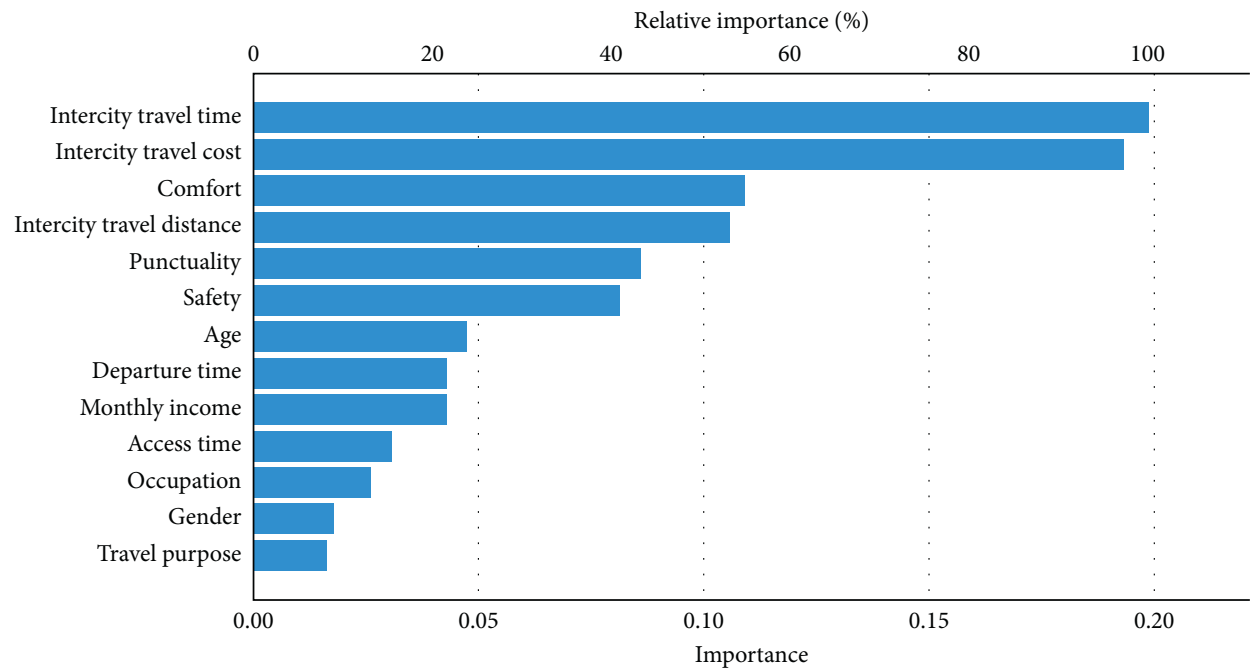

(b)

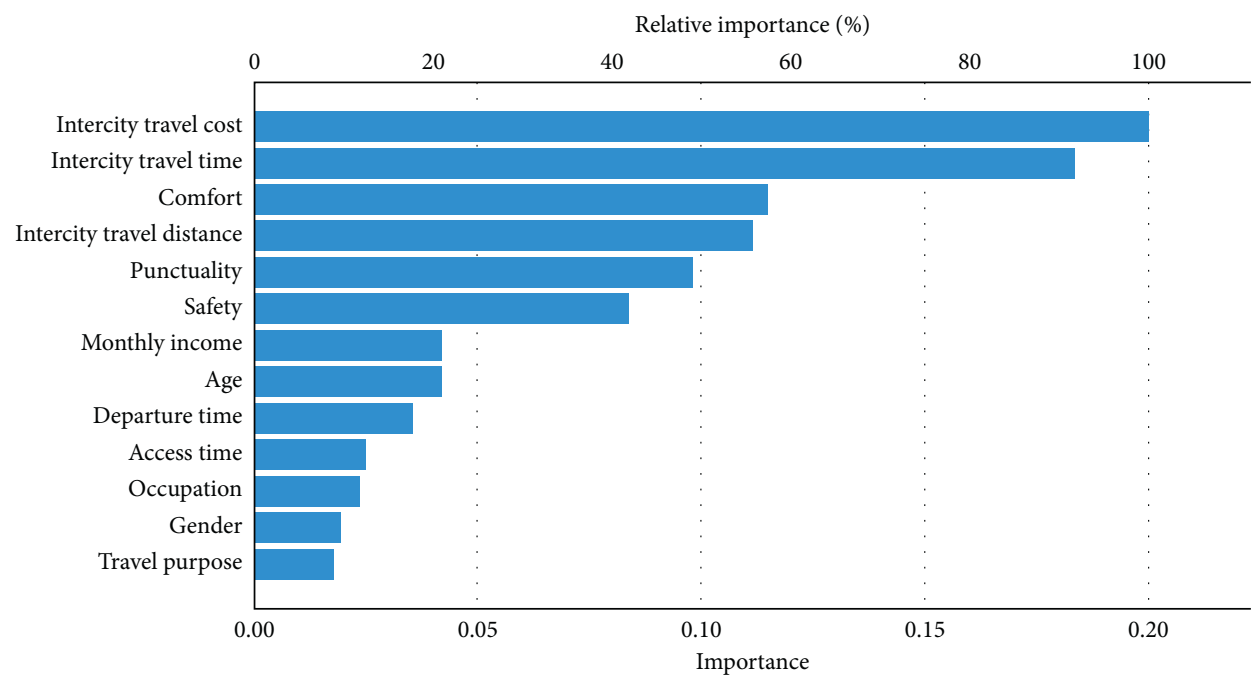

(c)

Figure 3: Relative importance of each variable using RBF. (a) Imbalanced data. (b) Oversampling balanced data. (c) Undersampling balanced data. 


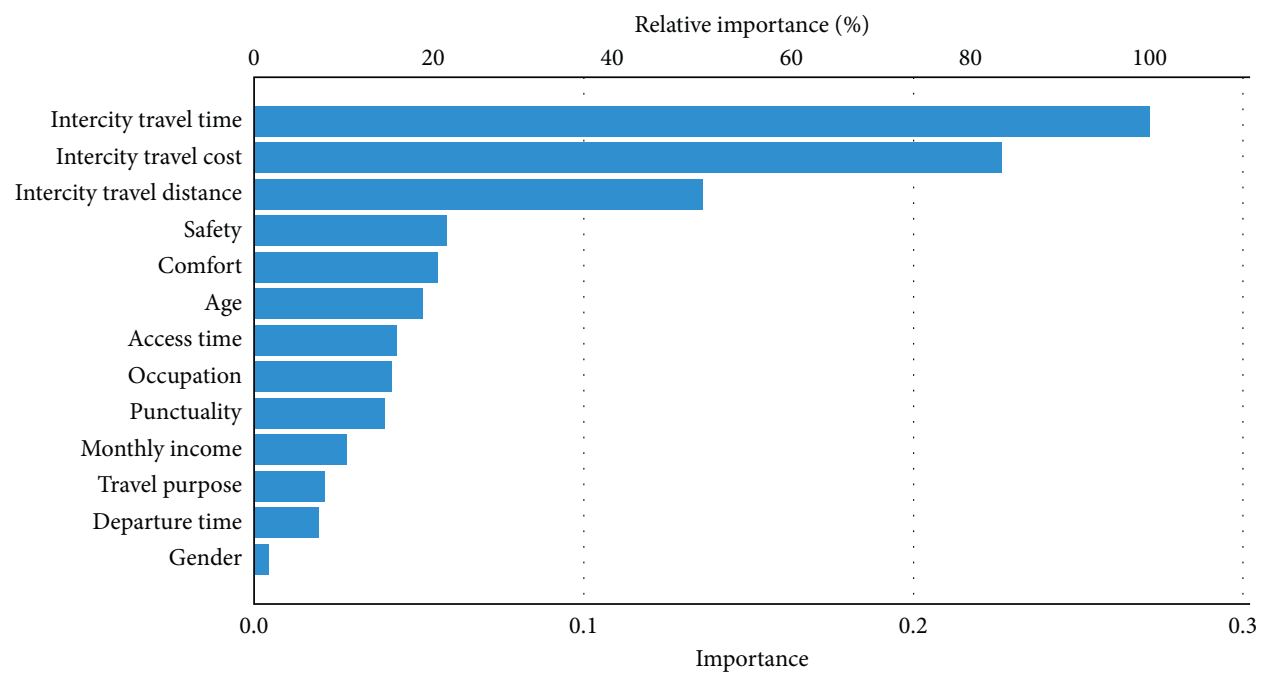

(a)

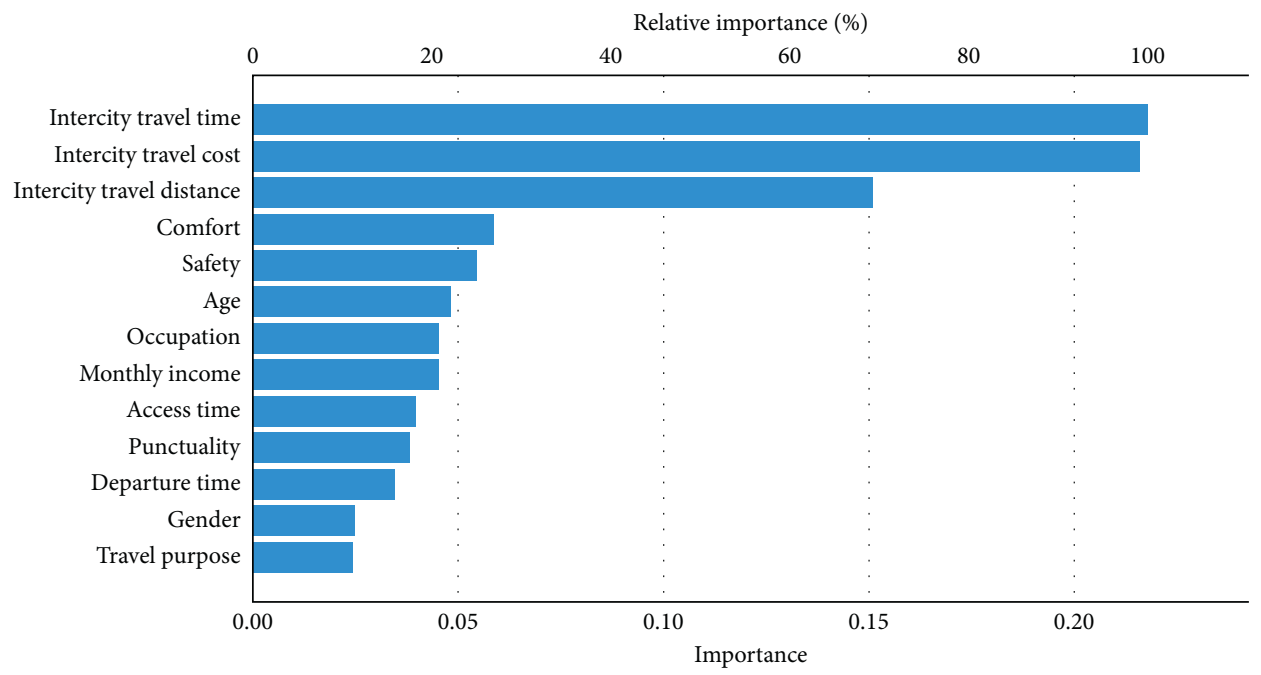

(b)

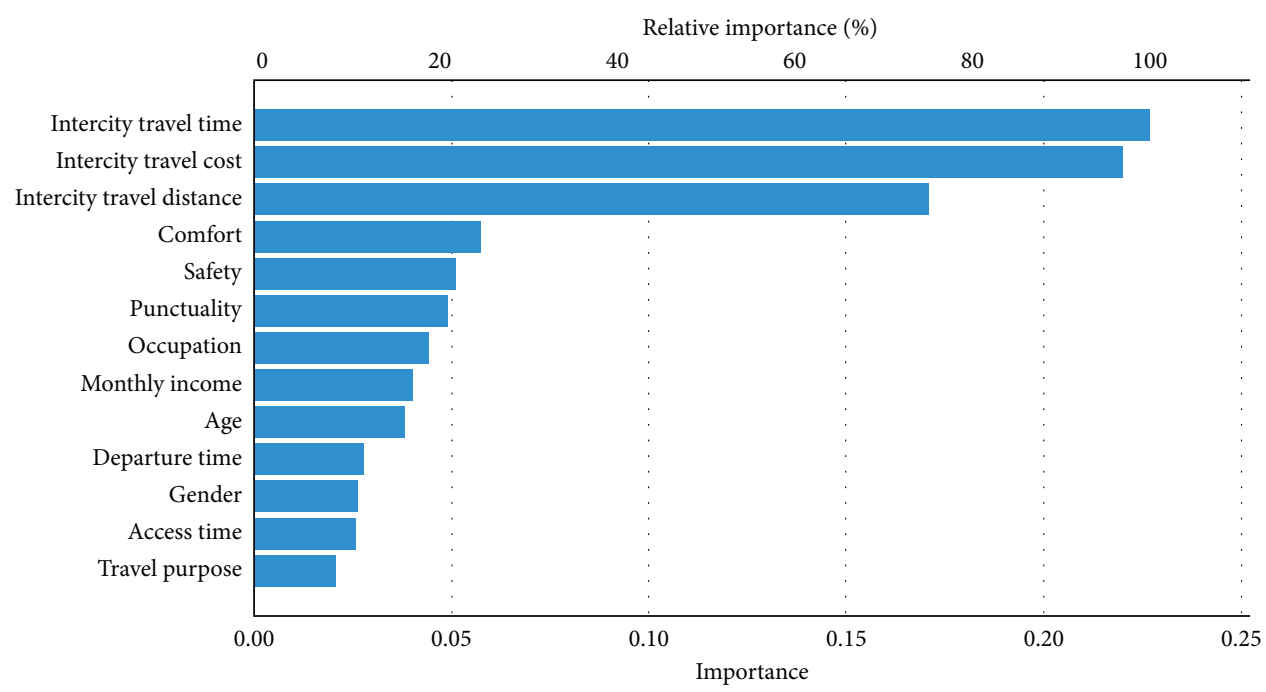

(c)

FIGURE 4: Relative importance of each variable using MLP. (a) Imbalanced data. (b) Oversampling balanced data. (c) Undersampling balanced data. 
TABLE 12: Confusion matrix and recall, precision, and accuracy of each model for imbalanced data.

\begin{tabular}{|c|c|c|c|c|c|c|c|c|c|c|c|c|}
\hline \multirow{3}{*}{ Model } & & \multicolumn{6}{|c|}{ Training set } & \multicolumn{5}{|c|}{ Predictive set } \\
\hline & & \multicolumn{6}{|c|}{ Predictive class } & \multicolumn{5}{|c|}{ Predictive class } \\
\hline & & Mode & Airplane & HSR & Train & Express bus & Recall & Airplane & HSR & Train & Express bus & Recall \\
\hline \multirow{5}{*}{ MNL } & \multirow{5}{*}{ Actual class } & Airplane & 109 & 18 & 2 & 0 & $84.50 \%$ & 30 & 2 & 0 & 0 & $93.80 \%$ \\
\hline & & HSR & 16 & 246 & 27 & 6 & $83.40 \%$ & 3 & 57 & 11 & 3 & $77.00 \%$ \\
\hline & & Train & 3 & 25 & 181 & 28 & $76.40 \%$ & 3 & 5 & 44 & 8 & $73.30 \%$ \\
\hline & & Express bus & 0 & 8 & 54 & 59 & $48.80 \%$ & 0 & 1 & 12 & 19 & $59.40 \%$ \\
\hline & & Precision & $85.16 \%$ & $82.83 \%$ & $68.56 \%$ & $63.44 \%$ & $76.10 \%$ & $83.33 \%$ & $87.69 \%$ & $65.67 \%$ & $63.33 \%$ & $75.76 \%$ \\
\hline \multirow{5}{*}{ BMNL } & \multirow{5}{*}{ Actual class } & Airplane & 113 & 14 & 2 & 0 & $87.60 \%$ & 30 & 2 & 0 & 0 & $93.80 \%$ \\
\hline & & HSR & 18 & 243 & 28 & 6 & $82.40 \%$ & 3 & 55 & 13 & 3 & $74.30 \%$ \\
\hline & & Train & 5 & 24 & 184 & 26 & $77.00 \%$ & 3 & 5 & 45 & 7 & $75.00 \%$ \\
\hline & & Express bus & 3 & 7 & 53 & 61 & $49.20 \%$ & 0 & 0 & 13 & 19 & $59.40 \%$ \\
\hline & & Precision & $81.29 \%$ & $84.38 \%$ & $68.91 \%$ & $65.59 \%$ & $76.36 \%$ & $83.33 \%$ & $88.71 \%$ & $63.38 \%$ & $65.52 \%$ & $75.25 \%$ \\
\hline \multirow{5}{*}{ MLP } & \multirow{5}{*}{ Actual class } & Airplane & 118 & 9 & 2 & 0 & $91.50 \%$ & 29 & 3 & 0 & 0 & $90.60 \%$ \\
\hline & & HSR & 12 & 268 & 13 & 2 & $90.80 \%$ & 3 & 64 & 6 & 1 & $86.50 \%$ \\
\hline & & Train & 1 & 30 & 183 & 23 & $77.20 \%$ & 0 & 7 & 45 & 7 & $76.30 \%$ \\
\hline & & Express bus & 0 & 5 & 54 & 62 & $51.20 \%$ & 0 & 2 & 13 & 17 & $53.10 \%$ \\
\hline & & Precision & $90.08 \%$ & $85.90 \%$ & $72.62 \%$ & $71.26 \%$ & $80.70 \%$ & $90.63 \%$ & $84.21 \%$ & $70.31 \%$ & $68.00 \%$ & $78.70 \%$ \\
\hline \multirow{5}{*}{$\mathrm{RBF}$} & \multirow{5}{*}{ Actual class } & Airplane & 80 & 41 & 6 & 2 & $62.00 \%$ & 21 & 11 & 0 & 0 & $65.60 \%$ \\
\hline & & HSR & 17 & 216 & 45 & 17 & $73.20 \%$ & 4 & 53 & 12 & 5 & $71.60 \%$ \\
\hline & & Train & 0 & 39 & 181 & 17 & $76.40 \%$ & 1 & 9 & 38 & 11 & $64.40 \%$ \\
\hline & & Express bus & 0 & 18 & 54 & 49 & $40.50 \%$ & 0 & 1 & 14 & 17 & $53.10 \%$ \\
\hline & & Precision & $82.47 \%$ & $68.79 \%$ & $63.29 \%$ & $57.65 \%$ & $67.30 \%$ & $80.77 \%$ & $71.62 \%$ & $59.38 \%$ & $51.52 \%$ & $65.50 \%$ \\
\hline
\end{tabular}

The bold values represent the accuracy of models.

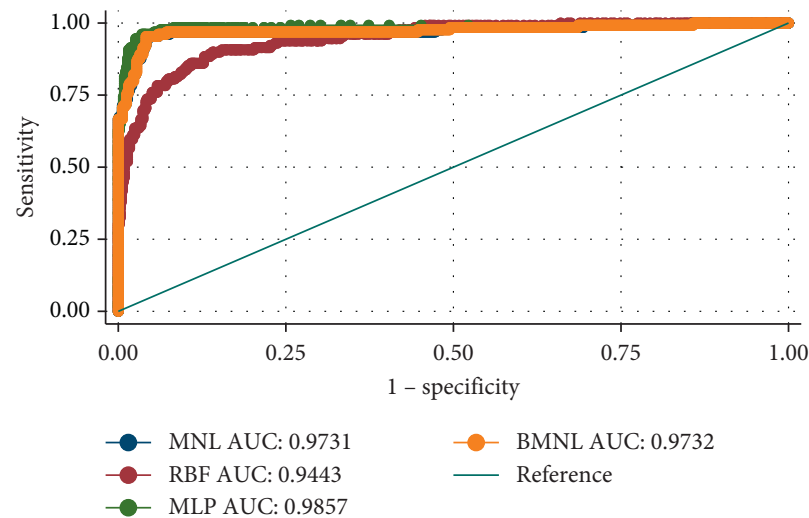

(a)

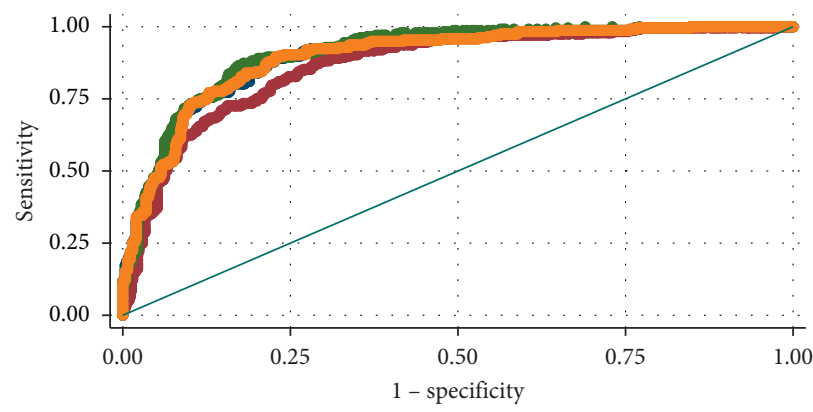

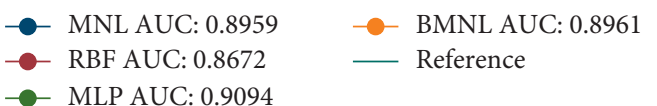

(c)

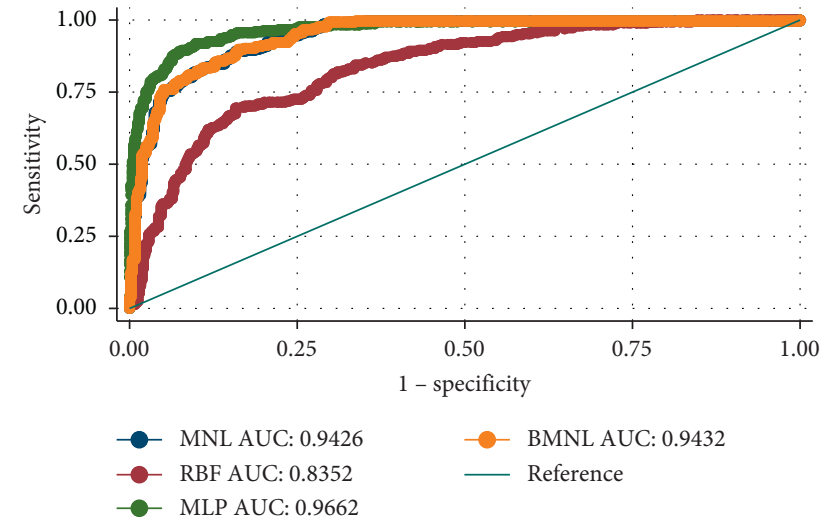

(b)

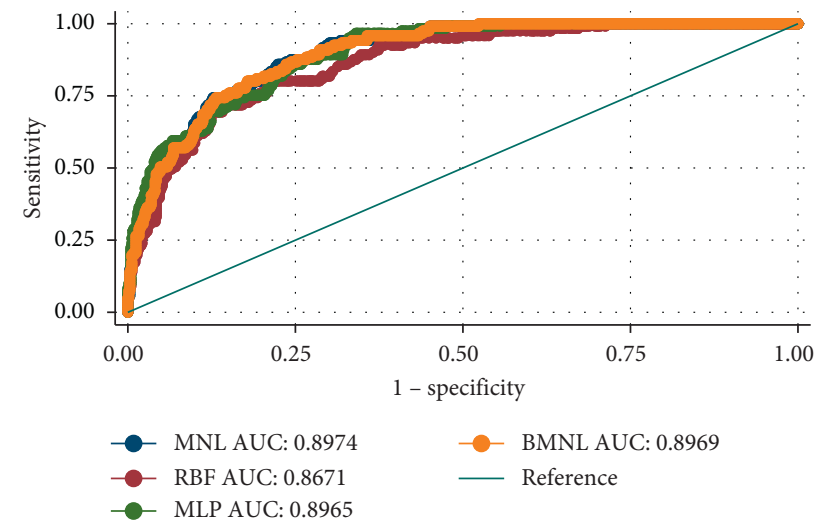

(d)

FIgURE 5: ROC curves of models for imbalanced data training set. (a) Airplane. (b) HSR. (c) Train. (d) Express bus. 


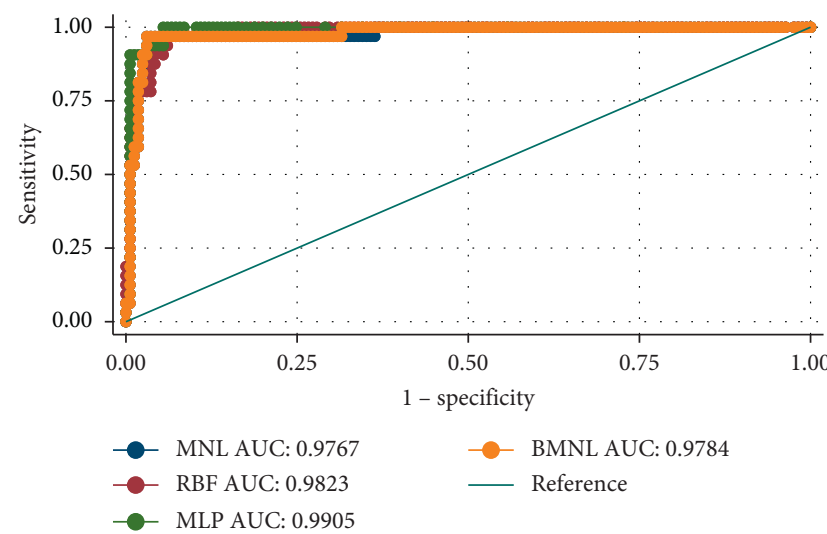

(a)

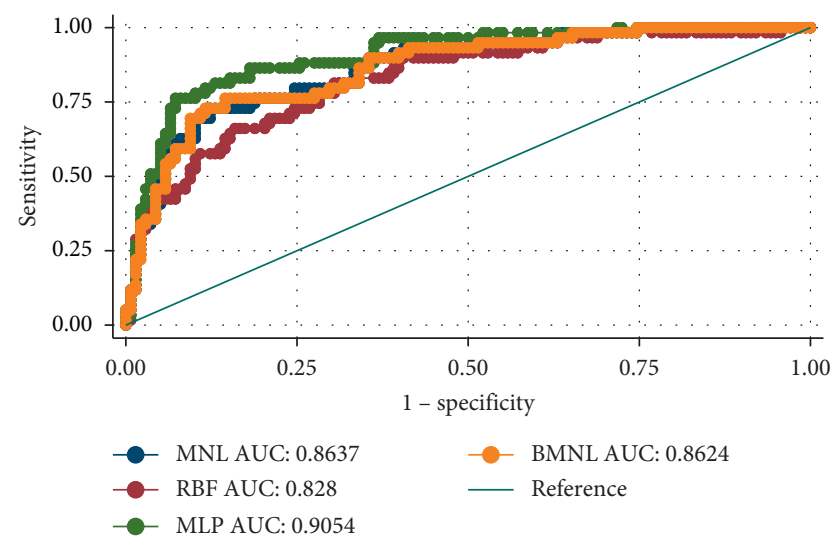

(c)

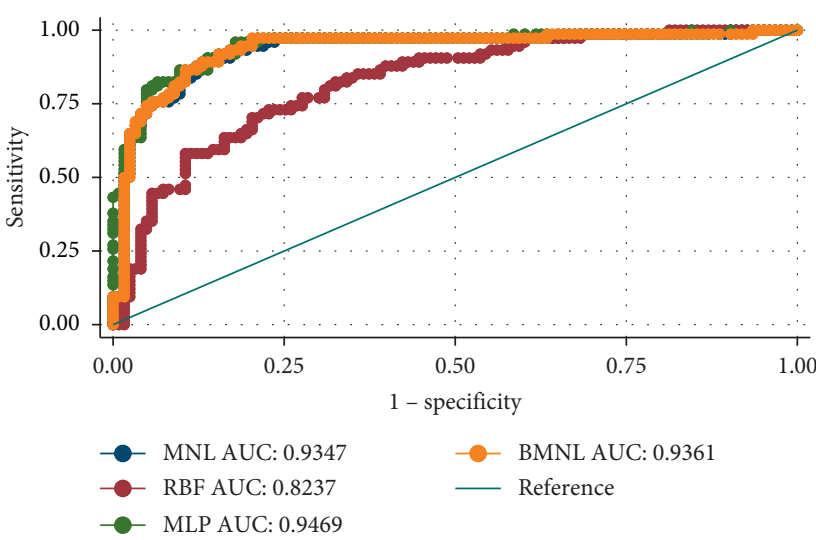

(b)

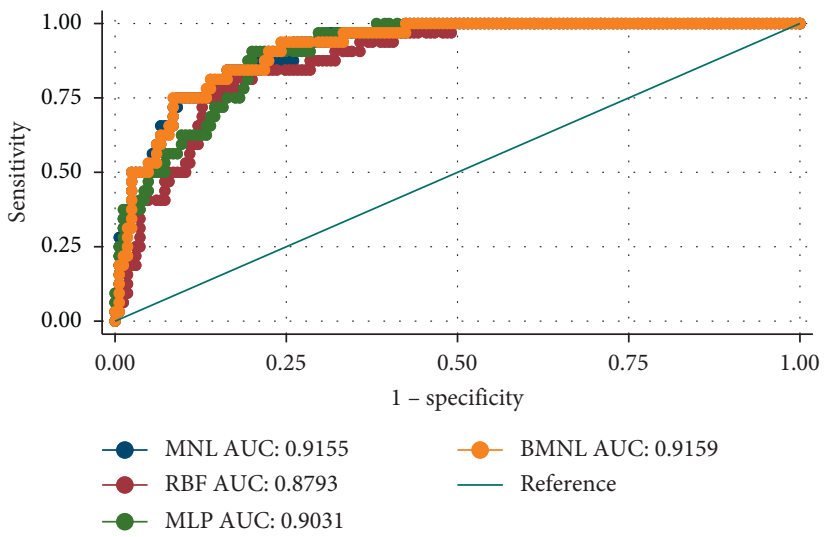

(d)

FIGURE 6: ROC curves of models for imbalanced data predictive set. (a) Airplane. (b) HSR. (c) Train. (d) Express bus.

TABLE 13: Confusion matrix and recall, precision, and accuracy of each model for undersampling of balanced data.

\begin{tabular}{|c|c|c|c|c|c|c|c|c|c|c|c|c|}
\hline \multirow{3}{*}{ Model } & & \multicolumn{6}{|c|}{ Training set } & \multicolumn{5}{|c|}{ Predictive set } \\
\hline & & \multicolumn{6}{|c|}{ Predictive class } & \multicolumn{5}{|c|}{ Predictive class } \\
\hline & & Mode & Airplane & HSR & Train & Express bus & Recall & Airplane & HSR & Train & Express bus & Recall \\
\hline \multirow{5}{*}{ MNL } & \multirow{5}{*}{ Actual class } & Airplane & 115 & 8 & 1 & 1 & $92.00 \%$ & 27 & 4 & 0 & 0 & $87.10 \%$ \\
\hline & & HSR & 8 & 102 & 7 & 8 & $81.60 \%$ & 5 & 21 & 1 & 4 & $67.74 \%$ \\
\hline & & Train & 3 & 15 & 79 & 28 & $63.20 \%$ & 0 & 4 & 15 & 12 & $48.39 \%$ \\
\hline & & Express bus & 3 & 3 & 25 & 94 & $75.20 \%$ & 0 & 1 & 8 & 22 & $70.97 \%$ \\
\hline & & Precision & $89.15 \%$ & $79.69 \%$ & $70.54 \%$ & $71.76 \%$ & $78.00 \%$ & $84.38 \%$ & $70.00 \%$ & $62.50 \%$ & $57.89 \%$ & $68.55 \%$ \\
\hline \multirow{5}{*}{ BMNL } & \multirow{5}{*}{ Actual class } & Airplane & 116 & 7 & 1 & 1 & $92.80 \%$ & 28 & 3 & 0 & 0 & $90.32 \%$ \\
\hline & & HSR & 12 & 88 & 14 & 11 & $70.40 \%$ & 5 & 18 & 3 & 5 & $58.06 \%$ \\
\hline & & Train & 3 & 6 & 81 & 35 & $64.80 \%$ & 0 & 2 & 17 & 12 & $54.84 \%$ \\
\hline & & Express bus & 3 & 0 & 28 & 94 & $75.20 \%$ & 0 & 1 & 8 & 22 & $70.97 \%$ \\
\hline & & Precision & $86.57 \%$ & $87.13 \%$ & $65.32 \%$ & $66.67 \%$ & $75.80 \%$ & $84.85 \%$ & $75.00 \%$ & $60.71 \%$ & $56.41 \%$ & $68.55 \%$ \\
\hline \multirow{5}{*}{ MLP } & \multirow{5}{*}{ Actual class } & Airplane & 121 & 2 & 1 & 1 & $96.80 \%$ & 28 & 3 & 0 & 0 & $90.30 \%$ \\
\hline & & HSR & 6 & 107 & 7 & 5 & $85.60 \%$ & 2 & 25 & 1 & 3 & $80.60 \%$ \\
\hline & & Train & 1 & 13 & 92 & 17 & $74.80 \%$ & 0 & 4 & 19 & 8 & $61.30 \%$ \\
\hline & & Express bus & 0 & 3 & 30 & 89 & $73.00 \%$ & 0 & 2 & 10 & 19 & $61.30 \%$ \\
\hline & & Precision & $94.53 \%$ & $85.60 \%$ & $70.77 \%$ & $79.46 \%$ & $81.80 \%$ & $93.33 \%$ & $73.53 \%$ & $63.33 \%$ & $63.33 \%$ & $73.40 \%$ \\
\hline \multirow{5}{*}{$\mathrm{RBF}$} & \multirow{5}{*}{ Actual class } & Airplane & 105 & 12 & 3 & 5 & $84.00 \%$ & 25 & 5 & 0 & 1 & $80.60 \%$ \\
\hline & & HSR & 20 & 79 & 15 & 11 & $63.20 \%$ & 3 & 20 & 3 & 5 & $64.50 \%$ \\
\hline & & Train & 1 & 12 & 83 & 27 & $67.50 \%$ & 0 & 2 & 22 & 7 & $71.00 \%$ \\
\hline & & Express bus & 1 & 9 & 28 & 84 & $68.90 \%$ & 0 & 2 & 8 & 21 & $67.70 \%$ \\
\hline & & Precision & $82.68 \%$ & $70.54 \%$ & $64.34 \%$ & $66.14 \%$ & $70.20 \%$ & $89.29 \%$ & $68.97 \%$ & $66.67 \%$ & $61.76 \%$ & $71.00 \%$ \\
\hline
\end{tabular}

The bold values represent the accuracy of models. 
TABLE 14: Confusion matrix of each model for oversampling of balanced data.

\begin{tabular}{|c|c|c|c|c|c|c|c|c|c|c|c|c|}
\hline \multirow{3}{*}{ Model } & & \multicolumn{6}{|c|}{ Training set } & \multicolumn{5}{|c|}{ Predictive set } \\
\hline & & \multicolumn{6}{|c|}{ Predictive class } & \multicolumn{5}{|c|}{ Predictive class } \\
\hline & & Mode & Airplane & HSR & Train & Express bus & Recall & Airplane & HSR & Train & Express bus & Recall \\
\hline \multirow{5}{*}{ MNL } & \multirow{5}{*}{ Actual class } & Airplane & 275 & 18 & 1 & 1 & $93.22 \%$ & 71 & 1 & 1 & 1 & $95.95 \%$ \\
\hline & & HSR & 18 & 235 & 23 & 19 & $79.66 \%$ & 9 & 55 & 5 & 5 & $74.32 \%$ \\
\hline & & Train & 6 & 23 & 190 & 76 & $64.41 \%$ & 1 & 10 & 40 & 23 & $54.05 \%$ \\
\hline & & Express bus & 7 & 8 & 63 & 217 & $73.56 \%$ & 0 & 1 & 27 & 46 & $62.16 \%$ \\
\hline & & Precision & $89.87 \%$ & $82.75 \%$ & $68.59 \%$ & $69.33 \%$ & $77.71 \%$ & $87.65 \%$ & $82.09 \%$ & $54.79 \%$ & $61.33 \%$ & $71.62 \%$ \\
\hline \multirow{5}{*}{ BMNL } & \multirow{5}{*}{ Actual class } & Airplane & 276 & 17 & 1 & 1 & $93.56 \%$ & 71 & 1 & 1 & 1 & $95.95 \%$ \\
\hline & & HSR & 18 & 235 & 22 & 20 & $79.66 \%$ & 9 & 54 & 6 & 5 & $72.97 \%$ \\
\hline & & Train & 6 & 23 & 194 & 72 & $65.76 \%$ & 1 & 10 & 41 & 22 & $55.41 \%$ \\
\hline & & Express bus & 7 & 5 & 73 & 210 & $71.19 \%$ & 0 & 0 & 28 & 46 & $62.16 \%$ \\
\hline & & Precision & $89.90 \%$ & $83.93 \%$ & $66.90 \%$ & $69.31 \%$ & $77.54 \%$ & $87.65 \%$ & $83.08 \%$ & $53.95 \%$ & $62.16 \%$ & $71.62 \%$ \\
\hline \multirow{5}{*}{ MLP } & \multirow{5}{*}{ Actual class } & Airplane & 277 & 16 & 1 & 1 & $93.90 \%$ & 72 & 0 & 1 & 1 & $97.30 \%$ \\
\hline & & HSR & 13 & 241 & 21 & 20 & $81.70 \%$ & 6 & 55 & 5 & 8 & $74.30 \%$ \\
\hline & & Train & 3 & 21 & 209 & 58 & $71.80 \%$ & 1 & 13 & 42 & 18 & $56.80 \%$ \\
\hline & & Express bus & 0 & 13 & 31 & 244 & $84.70 \%$ & 0 & 2 & 15 & 57 & $77.00 \%$ \\
\hline & & Precision & $94.54 \%$ & $82.82 \%$ & $79.77 \%$ & $75.54 \%$ & $83.10 \%$ & $91.14 \%$ & $78.57 \%$ & $66.67 \%$ & $67.86 \%$ & $76.40 \%$ \\
\hline \multirow{5}{*}{$\mathrm{RBF}$} & \multirow{5}{*}{ Actual class } & Airplane & 244 & 33 & 6 & 12 & $82.70 \%$ & 58 & 10 & 3 & 3 & $78.40 \%$ \\
\hline & & HSR & 51 & 176 & 37 & 31 & $59.70 \%$ & 14 & 43 & 8 & 9 & $58.10 \%$ \\
\hline & & Train & 2 & 37 & 188 & 64 & $64.60 \%$ & 2 & 15 & 41 & 16 & $55.40 \%$ \\
\hline & & Express bus & 2 & 18 & 72 & 196 & $68.10 \%$ & 0 & 3 & 18 & 53 & $71.60 \%$ \\
\hline & & Precision & $81.61 \%$ & $66.67 \%$ & $62.05 \%$ & $64.69 \%$ & $68.14 \%$ & $78.38 \%$ & $60.56 \%$ & $58.57 \%$ & $65.43 \%$ & $65.90 \%$ \\
\hline
\end{tabular}

The bold values represent the accuracy of models.

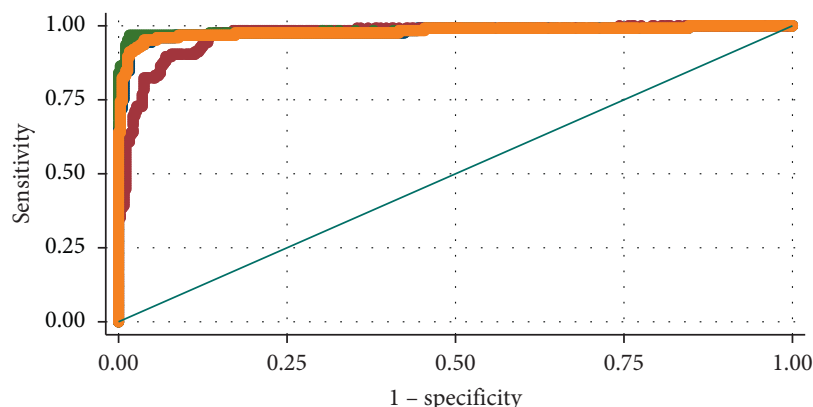

$\multimap \quad$ MNL AUC: 0.9806

$\rightarrow$ RBF AUC: 0.9671

$\rightarrow$ MLP AUC: 0.9855

(a)

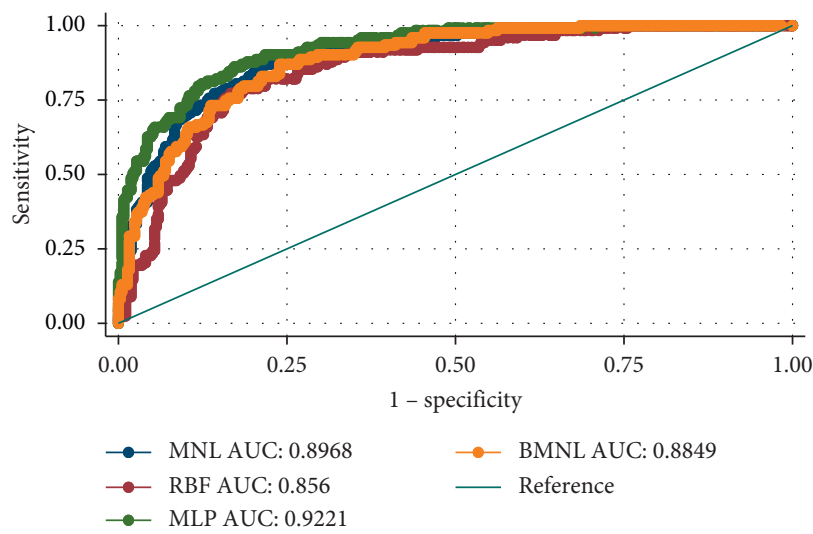

(c)

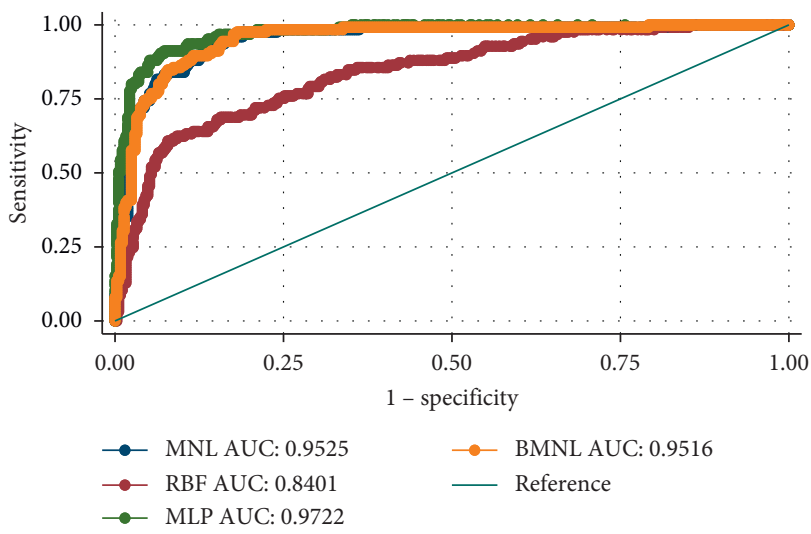

(b)

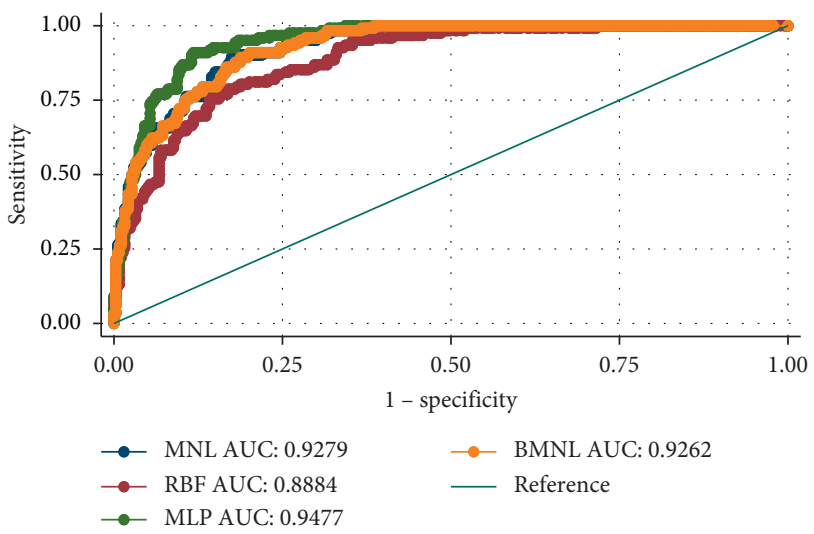

(d)

FIGURE 7: ROC curves for undersampled balanced data training set. (a) Airplane. (b) HSR. (c) Train. (d) Express bus. 


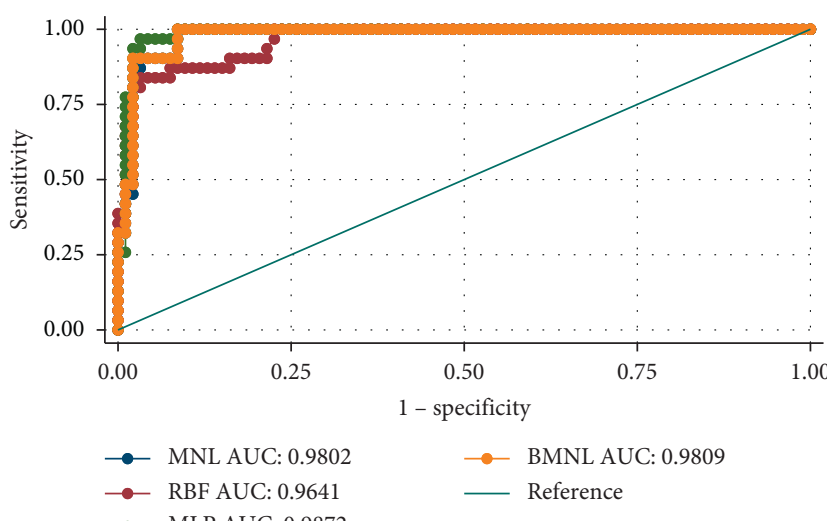

(a)

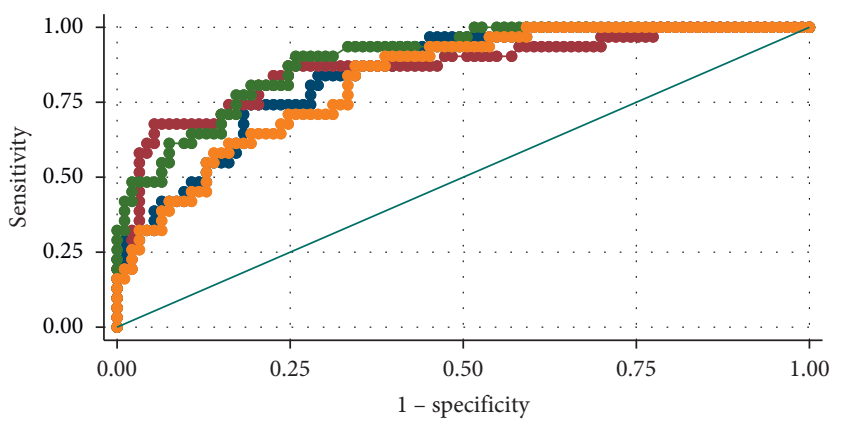

$\rightarrow$ MNL AUC: 0.8456

$\rightarrow$ RBF AUC: 0.8646

$\longrightarrow$ MLP AUC: 0.8909

(c)

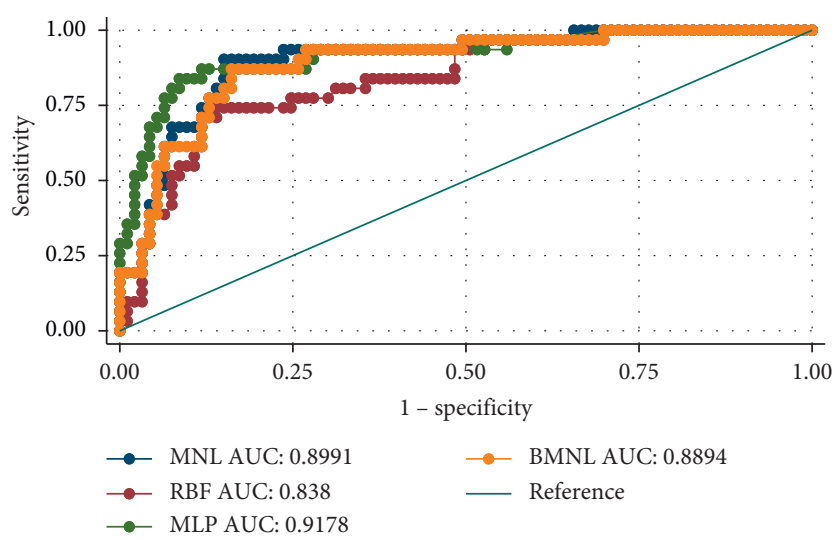

(b)

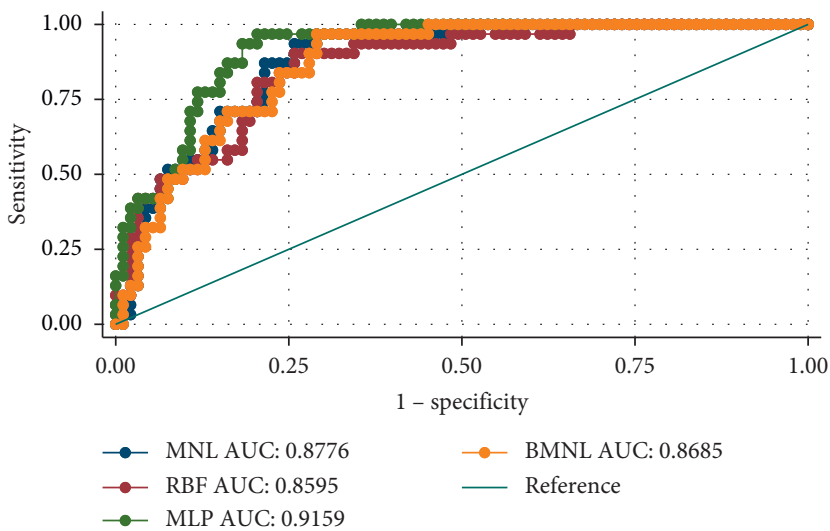

(d)

FigurE 8: ROC curves for undersampled balanced data predictive set. (a) Airplane. (b) HSR. (c) Train. (d) Express bus.

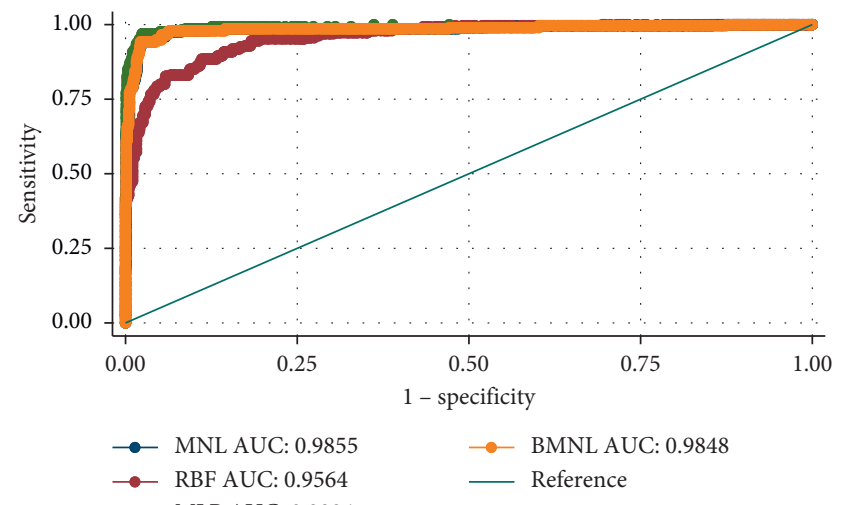

(a)

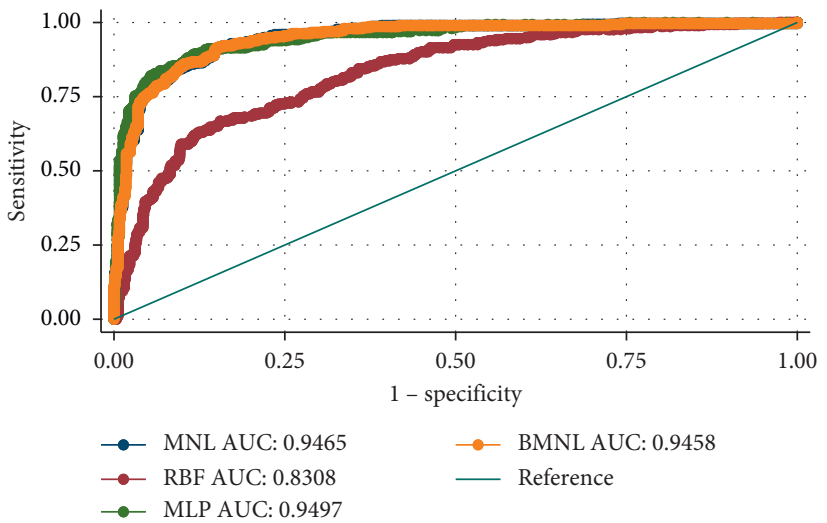

(b)

Figure 9: Continued. 


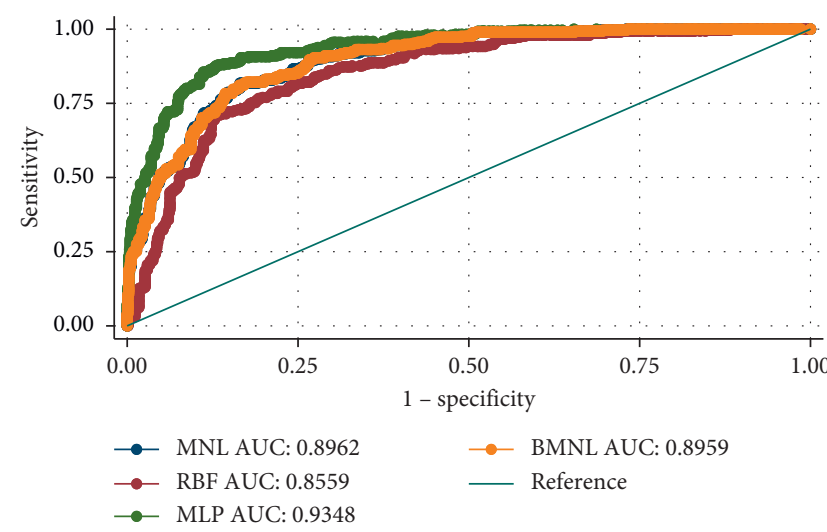

(c)

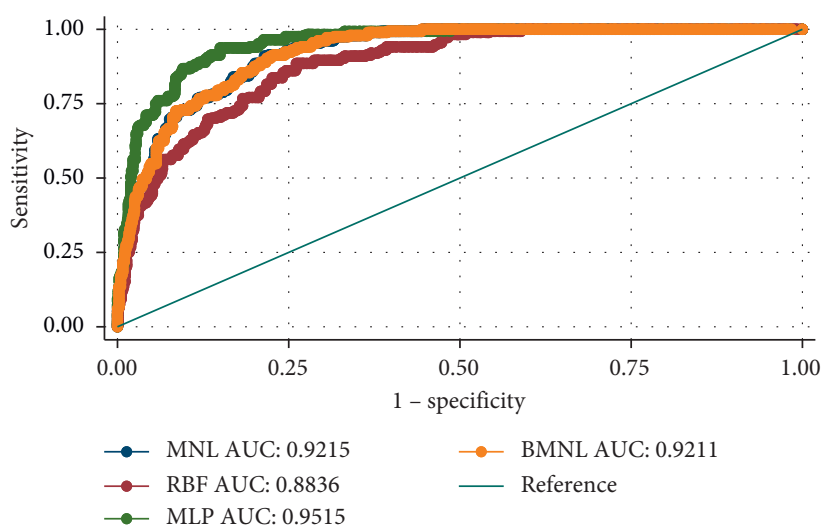

(d)

FIgURE 9: ROC curves for oversampled balanced data training set. (a) Airplane. (b) HSR. (c) Train. (d) Express bus.

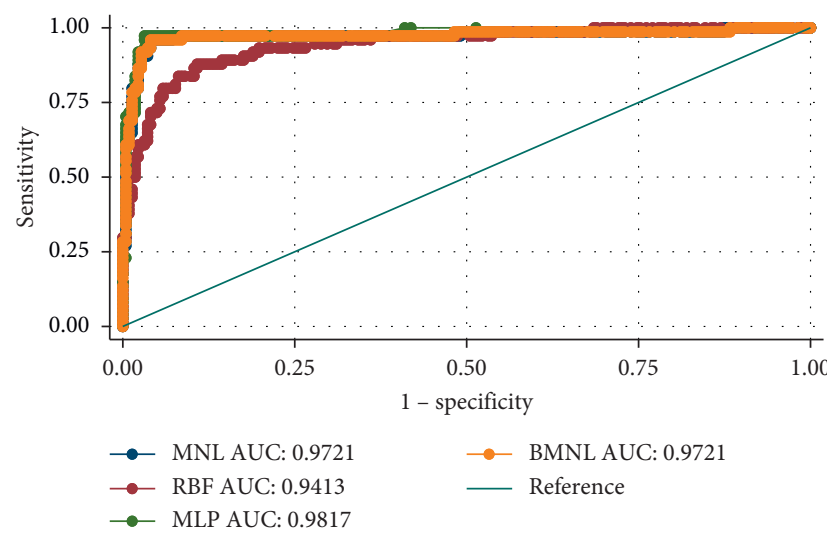

(a)

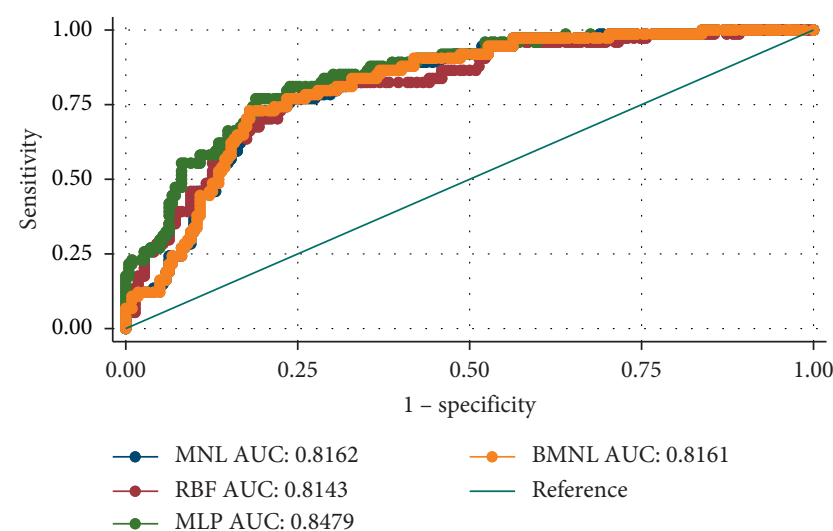

(c)

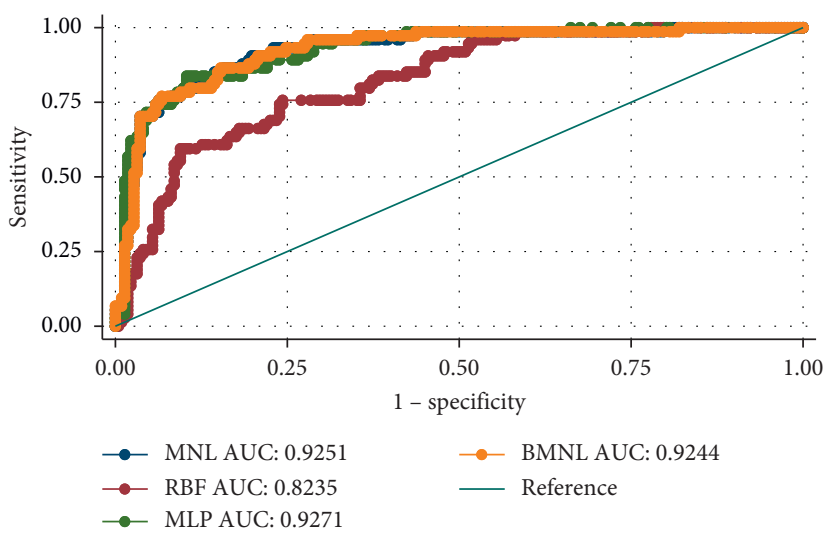

(b)

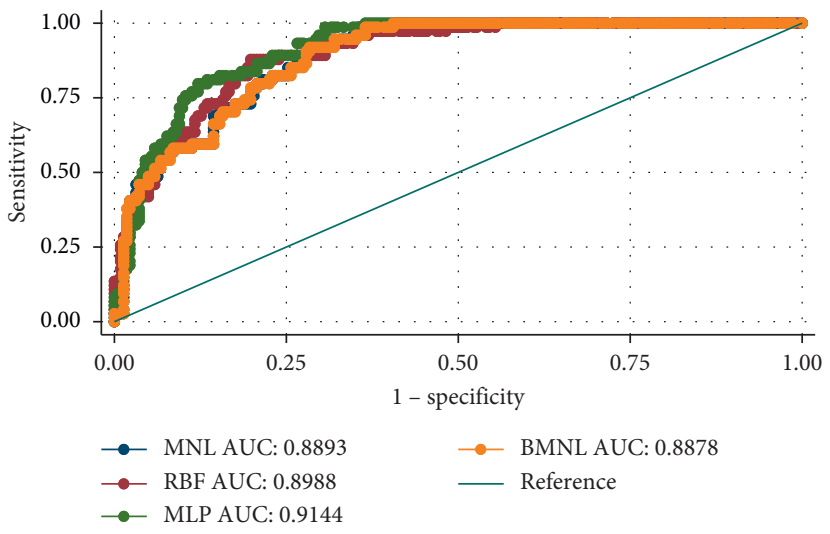

(d)

FIgURE 10: ROC curves for oversampled balanced data training set. (a) Airplane. (b) HSR. (c) Train. (d) Express bus.

with express bus choice. This result agrees with the result of a study $[4,7]$ that found that higher- and lower-income individuals favor air and bus travel, respectively.

The variable of travel purpose had a significant positive effect on HSR choice and was ranked 11th in the relative importance of all variables. This finding is similar to that of a past study [1] and reveals that, compared to the train, leisure passengers prefer HSRs or airplanes more than passengers for mandatory travel. It is possible that leisure passengers can afford the higher travel cost and are more willing to travel in a comfortable mode. The modeling results also show the significant impact of travel distance on 
intercity travel mode choice, which is third most important. This result implies that, compared to the train, the longer travel distance favors airplane and HSR, and the shorter distance favors express bus, which is consistent with previous studies $[3,10]$. Intercity travel cost is the most important variable and is a positive sign for the airplane or HSR choice, indicating that passengers incurring higher travel costs are more likely to travel by airplane or HSR. Intercity travel time is the second most important factor, showing a negative association with the choice of airplane and HSR, indicating that passengers spending less travel time are more likely to select airplane or HSR. This finding is intuitive because airplanes and HSRs are faster than trains.

Safety ranked fourth, and this variable affects the choice of airplane and HSR, showing that passengers with a higher safety demand are more likely to travel by airplane or HSR. Comfort is the fifth most important factor; it positively influences the choice of airplane and HSR and is negatively associated with express bus. This result is expected, as airplanes and HSRs have better service facilities and environments than trains [1]. Punctuality ranked ninth and is positively related to HSR choice and negatively associated with airplane and express bus. This shows that a higher punctuality demand favors HSR and does not favor airplane and express bus. This result is expected, as external conditions such as bad weather can easily affect the operation of airplanes and express buses, but its impact on HSRs and trains is relatively small [1].

Access time ranked seventh in relative importance and is found to have a positive effect on airplane choice and a negative effect on express bus compared to train, indicating that passengers spending longer access time prefer traveling by airplane and are less likely to travel by express bus. The finding is straightforward because the airport is generally farther than the railway station from the city center, and the highway passenger station is closer [10]. A similar result was found for the effect of departure time.

\section{Discussion and Conclusions}

We investigated modeling techniques BMNL, MNL, MLP, and RBF for passengers' intercity travel mode choices. Data from a large individual-level survey in the city of Xi' an were used to develop the model. More comprehensive factors such as socioeconomics, travel demand, service quality, and accessibility of transport hub were incorporated in the models.

The comparison results show that MLP has the best predictive performance, BMNL and MNL have approximately equal predictive accuracy, and RBF has the poorest performance using imbalanced data. It was found that the fitting performance of the four models with balanced data was slightly higher than those with imbalanced data. However, it was surprising that the predictive performance of these models with balanced data was slightly lower than those with imbalanced data. A potential reason could be that the degree of imbalance for the original data is very small. These findings suggest that the MLP and BMNL modeling approaches are recommended for the analysis of passengers' intercity travel mode choice. Significant variables in the BMNL model include gender, age, occupation, travel purpose, intercity travel distance, intercity travel cost, intercity travel time, safety, punctuality, access time, and departure time, which is not completely consistent with those in the MNL model. However, the signs of significant variables in the BMNL model were in line with those in the MNL model. Regarding the MLP modeling results, the travel cost was found to be the most important factor in intercity mode choice, followed by travel time and travel distance. Comfort, safety, and punctuality were relatively important factors for passenger travel mode choices. The influence of individual characteristics on intercity travel mode choices was relatively low, and monthly income was the most important factor among individual characteristics.

These findings can provide a reference for traffic management departments to formulate traffic demand management strategies and provide technical support for data analysts and high-tech enterprises to develop intelligent decision-making systems for the choice of passenger intercity travel modes. Through our research conclusion, we can find that intercity travel time, intercity travel cost, intercity travel distance, and the service quality of a transportation mode are important factors affecting intercity travel mode choices. Traffic transportation management departments can accordingly develop a green transportation development strategy by optimizing ticket prices, increasing vehicle speeds, and improving the quality of service, so as to push travelers from transportation with high energy consumption to that with low energy consumption. Our findings show that the predictive performance of models does not significantly improve when using balanced data instead of imbalanced data. This can provide a basis for data analysts to fully understand the impact of data structures on the predictive performance of models.

There are some limitations to this study. The results may only apply to the selected dataset and therefore must be verified using datasets from more cities. The degree of data imbalance and proportion between the training set and the prediction set may also affect the fitting and predictive performance of the models, and it is necessary to explore the fitting and predictive performance of models using extremely unbalanced data and other proportions in the future. In addition, although no significant multicollinearity was found in the independent variables for the models, intercity travel time and intercity travel cost varied with travel distance. It is necessary to generate the fare rate and intercity travel time per kilomile by standardizing the intercity travel time and intercity travel cost and incorporate the transformed variables into the models to eliminate the potential impact of travel distance. Moreover, more variables that might be associated with intercity travel mode choices, such as the characteristics of the destination city, weather, and coronavirus disease, should be investigated. Advanced modeling techniques, such as the Bayesian random parameter model capturing more unobserved heterogeneity, the Probit model with endogenous variables, and the XGBoost model, should be applied in future studies. 


\section{Data Availability}

The data used to support the findings of this study are available from the corresponding author upon request.

\section{Conflicts of Interest}

The authors declare that they have no conflicts of interest.

\section{Acknowledgments}

This work was supported by the National Natural Science Foundation of China (Grant no. 52002282), Shaanxi Natural Science Foundation Youth Project (Grant no. 2017JQ5086), Shaanxi Education Department Special Science and Technology Project Science (Grant no. 19JK0477), and Philosophy and Social Science Foundation of Zhejiang Province (Grant no. 21NDJC163YB).

\section{References}

[1] X. Li, J. Tang, X. Hu, and W. Wang, "Assessing intercity multimodal choice behavior in a touristy city: a factor analysis," Journal of Transport Geography, vol. 86, Article ID 102776, 2020.

[2] C. R. Bhat, "Accommodating variations in responsiveness to level-of-service measures in travel mode choice modeling," Transportation Research Part A: Policy and Practice, vol. 32, no. 7, pp. 495-507, 1998.

[3] V. V. Can, "Estimation of travel mode choice for domestic tourists to Nha Trang using the multinomial probit model," Transportation Research Part A: Policy and Practice, vol. 49, pp. 149-159, 2013.

[4] M. A. A. B. Miskeen, A. M. Alhodairi, and R. A. A. B. O. Rahmat, "Modeling a multinomial logit model of intercity travel mode choice behavior for all trips in Libya," International Journal of Civil \& Environmental Engineering, vol. 7, no. 9, pp. 636-645, 2013.

[5] S. Hess, G. Spitz, M. Bradley, and M. Coogan, "Analysis of mode choice for intercity travel: application of a hybrid choice model to two distinct US corridors," Transportation Research Part A: Policy and Practice, vol. 116, pp. 547-567, 2018.

[6] L. Cheng, X. Chen, J. De Vos, X. Lai, and F. Witlox, "Applying a random forest method approach to model travel mode choice behavior," Travel Behaviour and Society, vol. 14, pp. 1-10, 2019.

[7] C. V. Forinash and F. S. Koppelman, "Application and interpretation of nested logit models of intercity mode choice," Transportation Research Record: Journal of the Transportation Research Board, vol. 1413, no. 1413, pp. 98-106, 1993.

[8] C. R. Bhat, "An endogenous segmentation mode choice model with an application to intercity travel," Transportation Science, vol. 31, no. 1, pp. 34-48, 1997.

[9] Z.-C. Li and D. Sheng, "Forecasting passenger travel demand for air and high-speed rail integration service: a case study of Beijing-Guangzhou corridor, China," Transportation Research Part A: Policy and Practice, vol. 94, pp. 397-410, 2016.

[10] C. Román, J. C. Martín, R. Espino et al., "Valuation of travel time savings for intercity travel: the Madrid-Barcelona corridor," Transport Policy, vol. 36, pp. 105-117, 2014.

[11] Y. Guo, Z. Li, Y. Wu, and C. Xu, "Evaluating factors affecting electric bike users' registration of license plate in China using
Bayesian approach," Transportation Research Part F: Traffic Psychology and Behaviour, vol. 59, pp. 212-221, 2018.

[12] C. R. Bhat, "A heteroscedastic extreme value model of intercity travel mode choice," Transportation Research Part B: Methodological, vol. 29, no. 6, pp. 471-483, 1995.

[13] Y. Wang, L. Li, L. Wang, A. Moore, S. Staley, and Z. Li, "Modeling traveler mode choice behavior of a new high-speed rail corridor in China," Transportation Planning and Technology, vol. 37, no. 5, pp. 466-483, 2014.

[14] S. Ashiabor, H. Baik, and A. Trani, "Logit models for forecasting nationwide intercity travel demand in the United States," Transportation Research Record: Journal of the Transportation Research Board, vol. 2007, no. 1, pp. 1-12, 2007.

[15] J.-H. Lee, K.-S. Chon, and C. Park, "Accommodating heterogeneity and heteroscedasticity in intercity travel mode choice model: formulation and application to HoNam, South Korea, high-speed rail demand analysis," Transportation Research Record: Journal of the Transportation Research Board, vol. 1898, no. 1, pp. 69-78, 2004.

[16] Y. Guo, P. Liu, Y. Wu, and J. Chen, "Evaluating how rightturn treatments affect right-turn-on-red conflicts at signalized intersections," Journal of Transportation Safety \& Security, vol. 12, no. 3, pp. 419-440, 2020.

[17] S. Srinivasan, C. R. Bhat, and J. Holguin-Veras, "Empirical analysis of the impact of security perception on intercity mode choice," Transportation Research Record: Journal of the Transportation Research Board, vol. 1942, no. 1, pp. 9-15, 2006.

[18] X. Li, W. Wang, C. Xu, Z. Li, and B. Wang, "Multi-objective optimization of urban bus network using cumulative prospect theory," Journal of Systems Science and Complexity, vol. 28, no. 3, pp. 661-678, 2015.

[19] Y. Guo, Z. Li, Y. Wu, and C. Xu, "Exploring unobserved heterogeneity in bicyclists' red-light running behaviors at different crossing facilities," Accident Analysis \& Prevention, vol. 115, pp. 118-127, 2018.

[20] C.-X. Zhang, S. Xu, and J.-S. Zhang, “A novel variational Bayesian method for variable selection in logistic regression models," Computational Statistics \& Data Analysis, vol. 133, pp. 1-19, 2019.

[21] A. P. Afghari, M. M. Haque, S. Washington, and T. Smyth, "Effects of globally obtained informative priors on Bayesian safety performance functions developed for Australian crash data," Accident Analysis \& Prevention, vol. 129, pp. 55-65, 2019.

[22] X. Zhou, M. Wang, and D. Li, "Bike-sharing or taxi? Modeling the choices of travel mode in Chicago using machine learning," Journal of Transport Geography, vol. 79, 2019.

[23] M. Wong and B. Farooq, "A bi-partite generative model framework for analyzing and simulating large scale multiple discrete-continuous travel behaviour data," Transportation Research Part C: Emerging Technologies, vol. 110, pp. 247-268, 2020.

[24] X. Zhao, X. Yan, A. Yu, and P. Van Hentenryck, "Prediction and behavioral analysis of travel mode choice: a comparison of machine learning and logit models," Travel Behaviour and Society, vol. 20, pp. 22-35, 2020.

[25] C. Xie, J. Lu, and E. Parkany, "Work travel mode choice modeling with data mining: decision trees and neural networks," Transportation Research Record: Journal of the Transportation Research Board, vol. 1854, no. 1, pp. 50-61, 2003.

[26] Y. Zhang and Y. Xie, "Travel mode choice modeling with support vector machines," Transportation Research Record: 
Journal of the Transportation Research Board, vol. 2076, no. 1, pp. 141-150, 2008.

[27] Y. Wang, S. Peng, and M. Xu, "Emergency logistics network design based on space-time resource configuration," Knowledge-Based Systems, vol. 223, Article ID 107041, 2021.

[28] J. Hagenauer and M. Helbich, "A comparative study of machine learning classifiers for modeling travel mode choice," Expert Systems with Applications, vol. 78, pp. 273-282, 2017.

[29] Y. Wang, Y. Yuan, X. Guan et al., "Collaborative two-echelon multicenter vehicle routing optimization based on statespace-time network representation," Journal of Cleaner Production, vol. 258, Article ID 120590, 2020.

[30] F. Wang and C. L. Ross, "Machine learning travel mode choices: comparing the performance of an extreme gradient boosting model with a multinomial logit model," Transportation Research Record: Journal of the Transportation Research Board, vol. 2672, no. 47, 2018.

[31] M. Wong, B. Farooq, and G.-A. Bilodeau, "Discriminative conditional restricted Boltzmann machine for discrete choice and latent variable modelling," Journal of Choice Modelling, vol. 29, pp. 152-168, 2018.

[32] Y. Wang, S. Peng, X. Zhou, M. Mahmoudi, and L. Zhen, "Green logistics location-routing problem with eco-packages," Transportation Research Part E: Logistics and Transportation Review, vol. 143, Article ID 102118, 2020.

[33] A. Lindner, C. S. Pitombo, and A. L. Cunha, "Estimating motorized travel mode choice using classifiers: an application for high-dimensional multicollinear data," Travel Behaviour and Society, vol. 6, pp. 100-109, 2017.

[34] S. Rasouli and H. J. P. Timmermans, "Using ensembles of decision trees to predict transport mode choice decisions: effects on predictive success and uncertainty estimates," European Journal of Transport and Infrastructure Research, vol. 14, pp. 412-424, 2014.

[35] H. B. Celikoglu, "Application of radial basis function and generalized regression neural networks in non-linear utility function specification for travel mode choice modelling," Mathematical and Computer Modelling, vol. 44, no. 7-8, pp. 640-658, 2006.

[36] L. Wang and Z. Zuo, "Travel mode recognition using RBF neural network," CICTP 2014: Safe, Smart, and Sustainable Multimodal Transportation Systems, pp. 711-721, 2014.

[37] J. Sun, J. Sun, and P. Chen, "Use of support vector machine models for real-time prediction of crash risk on urban expressways," Transportation Research Record: Journal of the Transportation Research Board, vol. 2432, no. 2432, pp. 91-98, 2014.

[38] J. Xie and Z. Qiu, "The effect of imbalanced data sets on LDA: a theoretical and empirical analysis," Pattern Recognition, vol. 40, no. 2, pp. 557-562, 2007.

[39] A. D'Addabbo and R. Maglietta, "Parallel selective sampling method for imbalance and large data classification," Pattern Recognition Letters, vol. 62, pp. 61-67, 2015.

[40] W. A. Rivera and P. Xanthopoulos, "A priori synthetic oversampling methods for increasing classification sensitivity in imbalanced data sets," Expert Systems with Applications, vol. 66, pp. 124-135, 2016.

[41] V. García, J. S. Sánchez, A. I. Marqués, R. Florencia, and G. Rivera, "Understanding the apparent superiority of oversampling through an analysis of local information for classimbalanced data," Expert Systems with Applications, vol. 158, Article ID 113026, 2020.

[42] X. Li, R. Ma, Y. Guo, W. Wang, B. Yan, and J. Chen, "Investigation of factors and their dynamic effects on intercity travel modes competition," Travel Behaviour and Society, vol. 23, pp. 166-176, 2021.

[43] H. A. Klaiber and R. H. Von Haefen, "Do random coefficients and alternative specific constants improve policy analysis? An empirical investigation of model fit and prediction," Environmental and Resource Economics, vol. 73, pp. 75-91, 2011.

[44] J. H. Friedman, "Greedy function approximation: a gradient boosting machine," Annals of Statistics, vol. 29, no. 5, pp. 1189-1232, 2001.

[45] A. Goldstein, A. Kapelner, J. Bleich, and E. Pitkin, "Peeking inside the black box: visualizing statistical learning with plots of individual conditional expectation," Journal of Computational \& Graphical Statistics, vol. 24, no. 1, pp. 44-65, 2015.

[46] C. Molnar, Interpretable Machine Learning: A Guide for Making Black Box Models Explainable, Lulu, Morrisville, NC, USA, 2018.

[47] R. Hecht-Nielsen, "Kolmogorov's mapping neural network existence theorem," Proceedings of the International Conference on Neural Networks, vol. 3, pp. 11-13, 1987.

[48] S. V. Stehman, "Estimating standard errors of accuracy assessment statistics under cluster sampling," Remote Sensing of Environment, vol. 60, no. 3, pp. 258-269, 1997.

[49] C. Xu, W. Wang, P. Liu, and F. Zhang, "Development of a real-time crash risk prediction model incorporating the various crash mechanisms across different traffic states," Traffic Injury Prevention, vol. 16, no. 1, pp. 28-35, 2015. 